Cipango Cahiers d'études japonaises

23 | 2020

Charbon et communautés minières au Japon

\title{
Les parias des houillères du Chikuhō : assimilation ou surdiscrimination?
}

The Pariahs of the Coal Mines of Chikuhō: Assimilation or Over-Discrimination?

\section{Kaiko Miyazaki}

\section{(2) OpenEdition}

1 Journals

Édition électronique

URL : https://journals.openedition.org/cipango/3496

DOI : $10.4000 /$ cipango.3496

ISSN : 2260-7706

Éditeur

INALCO

\section{Édition imprimée}

Date de publication : 1 juillet 2020

ISBN : 9782858313426

ISSN : $1164-5857$

\section{Référence électronique}

Kaiko Miyazaki, « Les parias des houillères du Chikuhō : assimilation ou surdiscrimination ? ", Cipango [En ligne], 23 | 2020, mis en ligne le 16 juin 2020, consulté le 30 juin 2021. URL : http:// journals.openedition.org/cipango/3496 ; DOI : https://doi.org/10.4000/cipango.3496

\section{(c) (7) (8)}

Cipango est mis à disposition selon les termes de la Licence Creative Commons Attribution - Pas d'Utilisation Commerciale 4.0 International. 


\title{
Les parias des houillères du Chikuhō : assimilation ou surdiscrimination ?
}

\author{
The Pariahs of the Coal Mines of Chikuho: \\ Assimilation or Over-Discrimination?
}

Kaiko MiYAzAKI

CEJ, Inalco

La région du Chikuhō 筑豊 ${ }^{1}$, au nord du Kyūshū, fut, jusqu'aux années 1950, l'une des principales zones de production de charbon du Japon ${ }^{2}$. Par la suite, ses houillères furent dépassées par celles du bassin minier d'Ishikari, à Hokkaidō, alors que la production nationale de charbon entamait sa phase de déclin. Si la dernière mine du Chikuhō a fermé en 1976, cette région reste encore fortement associée à l'histoire du charbon, comme en témoigne la reprise fréquente, jusqu’à Tōkyō, du Tankōbushi 炭坑節, le chant des mineurs du Chikuhō, dans les fêtes religieuses du Bon.

1. Le Chikuhō n'ayant pas de limites administratives strictes, nous suivons l'usage des historiens japonais, qui se réfèrent à ses quatre « districts » (gun 郡), à savoir ceux de Kaho 嘉穗, Kurate 鞍手, Onga 遠賀 et Tagawa 田川, tous situés dans le département de Fukuoka.

2. Le Chikuhō concentrait $54 \%$ de la production nationale en 1902, 50 \% en 1921 et encore $31 \%$ en 1951. YADA, 2014, p. 34-75. 
Bénéficiant d'un éclairage en revanche bien plus modeste est le lien entre burakumin $^{3}$ et charbon dans cette région. Certes, le tabou autour de ces populations de parias demeure vivace dans le Japon contemporain. Comme le fait remarquer l'économiste Tokita Yoshihisa 戸木田嘉久 (1924), auteur d'ouvrages sur les houillères du Kyūshū ${ }^{4}$, l'historiographie d'après-guerre a relativement peu abordé cette question, notamment les recherches sur la classe ouvrière. Pourtant, les communautés buraku sont particulièrement présentes dans le Chikuhō et souvent situées à proximité des anciennes mines. Pour les rares historiens traitant précisément de ce sujet ${ }^{5}$, la présence dans les houillères de ces populations de parias est incontestable.

Est-ce là un simple hasard ? En nous appuyant sur les sources statistiques primaires disponibles ${ }^{6}$, et au regard des travaux des historiens japonais sur cette question, nous tenterons de montrer dans un premier temps qu'il existe bien un lien organique entre la présence buraku dans le Chikuhō et l'industrie du charbon, procédant essentiellement de facteurs historiques et sociaux.

3. Dénommés entre autres eta (souillés) ou hinin (non-humains), ces termes désignaient à l'époque Edo (1603-1868) tout un ensemble de populations exclues des catégories institutionnelles. Elles sont alors figées professionnellement dans des métiers réservés, considérés comme impurs, et spatialement dans des quartiers précis, avec une très forte endogamie. En dépit de l'émancipation de 1871, lors de la modernisation de Meiji (1868-1912), la ségrégation sociale et spatiale perdure. Les lieux où habitent ces populations sont alors progressivement appelés tokushu buraku (hameaux spéciaux), puis simplement buraku et leur habitants burakumin (gens des hameaux).

4. ToKiтA, 1992, p. 162.

5. Le pionnier dans ce domaine est certainement Mahara Tetsuo, avec « Chikuhō tankō to buraku no keisei » (1964), reproduit dans MAHARA, 1973, p. 67-86. Il est suivi par les travaux de Nagasue Toshio, et plus récemment, de Sakamoto Yüichi (cf. infra). Souvent sympathisants de la «Ligue d'émancipation des buraku » (Buraku Kaihō Dōmei 部落 解放同盟, ou BKD, fondée en 1955), les historiens de la question buraku s'orientent davantage vers l'étude de l'histoire de la Suiheisha (cf. infra) ou de l'époque d'Edo - et finalement assez peu sur l'histoire économique et sociale plus contemporaine, dont les enjeux sont souvent plus sensibles.

6. Essentiellement des enquêtes publiques, menées par des organismes gouvernementaux, de la Restauration de Meiji (1868) à l'après-guerre. 
Ce point nous conduira à traiter des modalités de prolétarisation des communautés buraku à la fin du XIX ${ }^{e}$ siècle, de pair avec l'industrialisation des houillères. En effet, ces parias se sont-ils fondus indistinctement dans la classe ouvrière émergente ? Nous verrons comment la discrimination a perduré au sein du prolétariat minier, avec différentes stratégies de gestion de ces populations par les groupes industriels. Nous utiliserons à cette fin les archives du mensuel Suihei $g e p p \bar{o}$ 水平月報 ${ }^{7}$, l'organe de la Suiheisha ${ }^{8}$ dans le Kyūshū, dont certains éléments peuvent indirectement fournir un assez bon tableau de la situation (relativement peu documentée) des burakumin dans les houillères du Chikuhō, au cours des années 1920.

Pour être complet, nous poserons enfin la question de la confrontation de cette réalité buraku au sein des mouvements ouvriers. Comment les syndicats de mineurs et le Parti communiste japonais ont-ils pris en compte la particularité de cette minorité dans leurs luttes, et géré la forte présence de la Suiheisha dans le Chikuhō ? Les archives du Suihei geppō ainsi que le devenir de ce journal et de ses responsables nous permettront de mettre en évidence la nature et l'évolution du rapport des groupes de défense des burakumin aux autres syndicats de mineurs - entre solidarité, alliance et subordination.

7.Zen Kyūshū Suiheisha 全九州水平社, Suihei geppō 水平月報 (Bulletin mensuel de la Suiheisha de Kyūshū), n 128, 1924-1927. Archives reproduites dans ZEN KYūshū SUIHEISHA, 1985, p. 1109.

8. La Suiheisha, «Société des niveleurs » (de son nom complet : Zenkoku Suiheisha 全 国水平社, abrégé en Zensui) a été fondée en 1922. Elle se pose en défenseur des droits des burakumin et lutte pour l'égalité avec le reste de la population japonaise. 


\section{Réalités et modalités de la présence burakumin dans les mines du Chikuhō}

\section{Les débuts du charbon et la présence eta dans le Chikubō à l'époque d'Edo}

L'une des premières mentions connues de l'extraction du charbon dans le Chikuhō date de la fin du XVII ${ }^{\text {e }}$ siècle $(1686)^{9}$. À cette époque, la houille aurait été utilisée comme substitut du bois de chauffage au sein des populations qui n'avaient pas les moyens d'en acheter. Pour Kaibara Ekiken 貝原益軒 (1630-1714), penseur confucéen ayant vécu à cette époque, les communautés qui exploitaient et vendaient le charbon appartenaient essentiellement à la classe du «vil peuple»(senmin 賤民), située au bas de l'échelle sociale ${ }^{10}$. L'interprétation de ce terme est alors large, mais les historiens Nagasue Toshio 永末十四雄 (1925-) et Mahara Tetsuo 馬原鉄男 (1930-1992) ${ }^{11}$ se demandent s'il ne s'agirait pas de populations assimilées aux eta 穢多 et hinin 非人 ${ }^{12}$. Pour d'autres, comme Aso Tatsuo 安蘓龍生, ce terme désignerait alors simplement des agriculteurs pauvres ${ }^{13}$. Au milieu de l'époque Edo, les métiers d'extraction et de vente de charbon, ainsi que son utilisation domestique, semblent être réservés dans tous les cas aux classes les plus pauvres des sociétés paysannes du Chikuhō. Des populations proches, dans la hiérarchie des valeurs, des eta et des hinin.

9. Certaines sources évoquent la date de 1587, mais Aso Tatsuo, directeur du Musée d'histoire et du charbon de Tagawa, les considère comme peu fiables. Aso, 1979, p. 57.

10. Kaibara Ekiken 貝原益軒, Yamato honzō 大和本草 (1709), cité par Saкамото, 1997 , p. 86.

11. Mahara, 1973, p. 71 ; Nagasue, 1979, n 15, p. 94. Nous remercions vivement M. Bernard Thomann pour la mise à disposition de cet article.

12. Les eta («souillés ») et hinin (« non humains ») correspondent pour l'époque d'Edo (1603-1868) aux populations de parias professionnellement cantonnés à des métiers réservés, alors souvent considérés comme impurs, et spatialement contraints de résider dans des quartiers à l'écart du reste de la population. L'usage du terme tokushu burakumin 特殊部落民 (《gens des hameaux spéciaux 》), puis simplement burakumin, date du début du $\mathrm{XX}^{\mathrm{e}}$ siècle. Pour plus de précisions, voir Sabouret, 1983.

13. Aso, 1979 , p. 58. 
Le charbon devient cependant une ressource de plus en plus lucrative au XVIII ${ }^{\mathrm{e}}$ siècle, notamment dans le cadre de la fabrication du sel (enden 塩田), qui consomme de grandes quantités de bois, avantageusement remplacé par la houille lorsque celle-ci est disponible. L'extraction et le commerce du charbon passent alors dans les mains des agriculteurs, sous le contrôle de communautés villageoises employant des eta comme main-d'œuvre d'appoint. C'est le cas par exemple des paysans du village de Nakahara, dans le district de Kasuya (粕屋 郡), qui contrôlaient ces activités et firent appel, en 1782-1783, à des eta pour s'acquitter du travail ingrat consistant à vidanger l'eau des mines au jour de l'an ${ }^{14}$.

D'autres exemples montrent également que ces eta, exclus des activités charbonnières les plus lucratives comme l'extraction et la vente, s'occupaient surtout de travaux marginaux, dans des environnements difficiles. Ainsi, ils se consacraient notamment aux transports par voie d'eau, d'autant que les communautés eta étaient souvent installées près des rivières. Dans le Chikuhō, ils avaient en charge le transport du charbon sur les cours supérieurs des affluents de la rivière Onga, en tant que «bateliers » (kako 水主). Les eta utilisaient pour ce faire des embarcations adaptées aux caractéristiques de ces rivières, nommées takasebune 高瀬舟 dans la région de Tagawa, ou kawahirata 川艊 dans le Chikuzen. Les hyakushō 百姓 ${ }^{15}$, eux, s'occupaient du transport dans les cours inférieurs. On peut citer l'exemple d'un hameau buraku nommé Sendōmachi 船 頭町, le « bourg des bateliers », situé dans le district de Tagawa (fief de Kokura), sur la Chūganji, un affluent de l'Onga, et dont l'activité est attestée en 1852. On y trouve aussi les buraku de Gakide, de Toishi, de Hichijikkoku et de Miyatoko, tous chargés du transport fluvial du charbon. Mahara Tetsuo, recueillant les témoignages de burakumin qui avaient conservé les kawabirata de leurs ancêtres, considère que nombre d'entre eux travaillaient comme bateliers du charbon ${ }^{16}$.

14. Nagasue, 1979, p. 97.

15. Si la définition du terme hyakushō évolue selon les époques, celui-ci désigne durant l'époque d'Edo les roturiers habitant les zones rurales (mura 村), composées essentiellement de paysans, par opposition aux chōnin 町人 qui résident dans les zones urbaines (machi 町).

16. MAHARA, 1973 , p. 74. 
Pour Aso Tatsuo, leur contribution massive à ce secteur d'activité ne daterait en revanche que du début de l'ère Meiji (1868-1912), suite à la libéralisation du transport fluvial du charbon en 1872. Elle se serait poursuivie jusqu'aux années 1890 , où ils furent progressivement remplacés par le chemin de fer ${ }^{17}$.

L'intérêt accru porté au charbon dans le Chikuhō du XviII ${ }^{\mathrm{e}}$ siècle avait conduit les fiefs de Fukuoka et de Kokura à prendre en main sa gestion et son contrôle. Sans perdre de vue l'aspect lucratif de cette activité, ils privilégièrent cependant l'agriculture et tentèrent de faire revenir dans les rizières les hyakushō partis se consacrer au charbon. Ainsi, en 1803, le fief de Kokura en vint à interdire l'exploitation de la houille aux hyakushō, autorisant officiellement les « gens d'ailleurs » (yosomono 他所者) à travailler dans les mines. Durant la première moitié du $\mathrm{XIX}^{\mathrm{e}}$ siècle, la majeure partie de la main-d'œuvre charbonnière de la région est ainsi constituée de yosomono. Ce terme est parfois remplacé dans les documents d'époque par celui de 《bannis »(aburemono 溢者), ou plus simplement de « voyageurs » (tabibito 旅人), soulignant leur nomadisme et leur non-appartenance à la population générale. Ils sont également désignés selon la nature de leur travail par l'expression générique de «travailleurs journaliers » (biyatoi kasegi 日雇挊) ou, plus spécifiquement, par celle de « travailleurs des mines »(ishiyama kasegi 石山稼), voire de «gens des mines de charbon » (sekitan yama no mono 石炭山の者). La plupart d'entre eux n'est pas enregistrée dans l'état-civil de l'époque, et leur origine est rarement connue ${ }^{18}$. Endō Masao, économiste de l'université de Kyūshū, estimait en 1942 qu'il y avait aussi des eta et des hinin parmi ces vagabonds qui travaillaient dans les houillères ${ }^{19}$.

17. Aso, 1979, p. 77-79.

18. Ibid., p. 59-84.

19. Il affirme ainsi : « $\triangle \triangle[s i c]$ et hinin furent traités à l'époque Edo comme des classes du "vil peuple spécial", et il est probable qu'ils aient compté parmi les vagabonds qui travaillaient dans les mines de charbon pour s'y cacher » (Tokugawa jidai no tokushumin to shite toriatsukawareta kaikyū ni $\triangle \triangle$ [sic] oyobi hinin ga atta. Furōsha to shite tankō ni hairikonda mono no naka ni wa, korera no kaikyū mo attarō to omowareru 徳川時代の 特殊賤民として取扱はれた階級に $\triangle \triangle$ 及び非人があった。浮浪者として炭 坑に入り込んだものの中には、是等の階級もあつたらうと思われる [...]). Le symbole « $\triangle \triangle \gg$ remplace le mot eta. ENDō, 1942, p. 156-157. 
Pour Aso Tatsuo, ces mineurs sont surtout des hyakushō déchus en provenance d'autres régions du Japon, bien qu'il existe selon lui de grandes similitudes entre le statut des « gens des mines de charbon » et des eta : tous deux, en effet, n'ont pas le droit d'entrer dans les villages de hyakushō, et sont presque toujours jugés responsables des conflits pouvant les opposer à ces derniers, alors qu'ils seraient aujourd'hui plutôt considérés comme des victimes ${ }^{20}$.

Il est en revanche fort possible qu'une partie de ces mineurs-vagabonds ait été progressivement intégrée à la catégorie des eta. Dans le cas du Chikuhō, et contrairement à certaines parties du Japon, c'est surtout au sein des eta, et non des hinin, que les vagabonds semblent avoir été incorporés ${ }^{21}$ : le nombre de hinin est en effet particulièrement faible dans les deux fiefs de Fukuoka et de Kokura ${ }^{22}$, sans doute car la classe des eta répondait davantage aux besoins en main d'œuvre agricole stable.

Cette intégration aux eta pouvait également se produire après les famines meurtrières dues aux mauvaises récoltes, notamment la Grande Famine de l'ère Kyōhō, en 1732. Ces évènements provoquaient une hécatombe parmi les populations paysannes : la seule famine de 1732 a ainsi causé la mort de 43547 individus dans le fief de Kokura ${ }^{23}$, quand celui de Fukuoka vit disparaître $26 \%$ de sa population. Les survivants tendaient en outre à abandonner progressivement leurs terres, fuyant des impôts devenus de plus en plus lourds. Ainsi, à Tagawa, le nombre d'agriculteurs (non eta) était passé entre 1768 et 1852 de 31392 à 26124 individus $^{24}$.

Pour remédier à ce manque de main-d'œuvre, les deux fiefs firent d'abord appel à leurs propres populations eta en les affectant à la culture des rizières abandonnées dans le cadre des mesures d' « installation des nouveaux paysans »

20. Aso, 1979, p. $62-63$.

21. HARADA, 1975 [1973], p. 124-126.

22. En 1868, il n'y avait en effet aucun hinin dans le fief de Fukuoka pour 21485 eta, et seulement 82 hinin pour 6356 eta dans celui de Kokura. Chūō YŪWA JigYō KYŌKAI, 1974 [1939], p. 77-98.

23. Matsuzaki, 1979, p. 100.

24. Aso, 1979, p. 82. 


\section{CIPANGO}

70 Cahiers d'études japonaises $n^{\circ} 23$

(shinbyakushō shisue 新百姓仕据) menées dans la région au cours de la deuxième moitié du XVIII ${ }^{\mathrm{e}}$ siècle. Ils les installèrent ainsi en tant que « nouveaux paysans d'origine eta ( eta shinbyakushō 穢多新百姓). Afin d'augmenter en nombre cette main-d'œuvre agricole de rang eta, le fief de Kokura décréta en 1801 que les paysans ayant abandonné leurs terres seraient désormais intégrés d'office à la catégorie des eta, ce qui devait permettre par la suite de les contraindre à cultiver leurs parcelles ${ }^{25}$. Pendant cette période, on observe dans le fief une forte augmentation de la proportion d'eta par rapport à l'ensemble de la population, de $0,02 \%$ en 1622 à $5,4 \%$ en 1868 , alors que le taux de hinin stagnait toujours à $0,07 \%$ cette même année $1868^{26}$. Si l'on s'intéresse au seul district de Tagawa, on constate que l'augmentation démographique des eta correspond à une baisse du nombre de paysans : le nombre de foyers eta progresse en effet de 484 à 755 foyers entre 1818 et 1852 , quand les foyers paysans ${ }^{27}$, de 6333 en 1818, ne sont plus que 6124 en 1852.

25. TAKano, 2007.

26. CHŪō YŪWA JigYō KYŌKAI, 1974 [1939], p. 77-101\& nº 58, p. 101.

27. Il s'agit plus précisément du nombre de non eta, constitué essentiellement de paysans. Aso, 1979 , p. $57 \& 82$. 


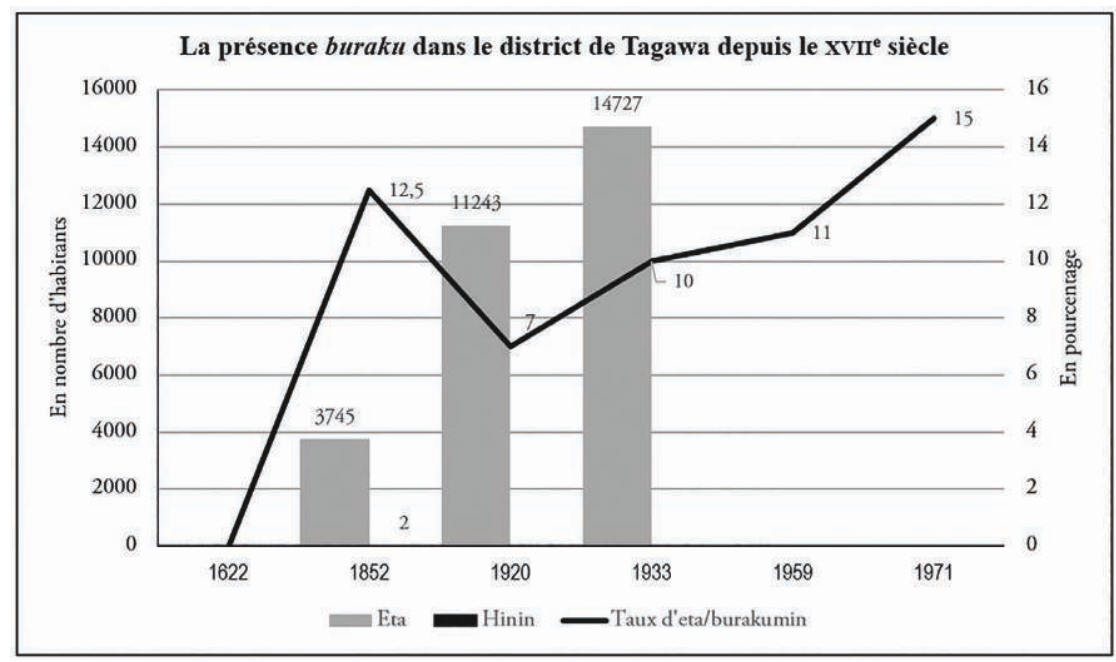

FIGURE 1. ÉVOLUTION DE LA PRÉSENCE ETA/HININ/BURAKUMIN

DANS LE DISTRICT DE TAGAWA ENTRE 1622 ET 1971.

Pour les années 1959 et 1971, le nombre de burakumin n'est pas disponible au niveau du district ${ }^{28}$.

Dans le fief de Fukuoka, où une politique similaire fut pratiquée, le nombre de villages eta connut de même une forte progression en deux siècles et demi, de 27 villages en 1604, à 133 en $1847^{29}$. Avec ces taux particulièrement élevés d'eta, les deux fiefs finirent par se distinguer nettement de leurs voisins : d'après l'enquête publique menée en 1868 , le taux d'eta s'élevait ainsi à 5,9\% dans le fief de Fukuoka et 5,4\% dans celui de Kokura, alors qu'il n'était que de 0,8 \%

28. Matsuzaki, 1979, p. 89 ; Sakamoto, 1997, p. 84 ; Aso, 1979, p. 57. Nous avons compilé des chiffres provenant de sources différentes selon l'époque : archives du fief de Kokura pour 1622, archives privées pour 1852, et quotidien Fukuoka Nichi.nichi 福 岡日日 pour 1920. S'ils ne peuvent être considérés comme d'une parfaite exactitude, les ordres de grandeur, leur adéquation avec la tendance générale et les auteurs qui les utilisent leurs confèrent néanmoins une certaine crédibilité.

29. Nagasue, 1989 , p. 72-75. 
72 Cahiers d'études japonaises nº 23

à Kurume, $0,7 \%$ à Yanagawa, $0 \%$ à Senzoku, et $1 \%$ pour Kumamoto ${ }^{30}$, soient des valeurs proches de celles qu'enregistraient à l'origine les fiefs de Fukuoka et Kokura.

Si l'augmentation démographique des eta dans ces deux fiefs a résulté du besoin de maintenir des revenus agricoles après les famines, il nous semble toutefois difficile d'omettre l'influence concomitante du développement des activités d'extraction du charbon dans la région. L'autorisation d'exploitation du charbon par les populations errantes, ainsi que leur installation dans de nouveaux bourgs, ont certainement eu un effet d'appel envers les travailleurs-migrants du Kyūshū, voire du reste du Japon. Ces mineurs-voyageurs pourraient ensuite avoir été intégrés à la catégorie des eta agriculteurs, surtout s'ils étaient souvent, comme l'affirme Aso Tatsuo, à l'origine des hyakushō. De plus, lorsqu'ils n'étaient pas en activité dans les rizières, les métayers travaillaient également dans les houillères en tant que journaliers. Cette hypothèse paraît particulièrement plausible dans le cas de Tagawa, au cœur des régions productrices de charbon. Le district voit en effet augmenter le nombre de ses eta de façon spectaculaire entre le XVII ${ }^{\mathrm{e}}$ et le XIX ${ }^{\mathrm{e}}$ siècle, de $0 \%$ de sa population en 1622 , à $12,5 \%$ en 1852 , soit plus du double de la moyenne du fief de Kokura (figure 1).

On retrouve par ailleurs dans les villages eta du district de Tagawa les traces d'une forte immigration. Ainsi, dans les villages de Miyao, Kamiyugeta, Shimoyugeta et Kawarayugeta, $16 \%$ des habitants eta de 1852 s'avèrent être originaires d'un autre district, ou d'un autre fief que celui de Kokura ${ }^{31}$. Cette immigration extra-seigneuriale ressemble fortement au profil des mineurs yosomono et tabibito. Elle conforte l'idée que ceux-ci ont bien été intégrés à la catégorie eta, et confirme aussi l'importance des liens entre activité minière et présence eta dans le Chikuhō. Cette immigration, avec le renfort des métayers établis par les fiefs comme « nouveaux paysans d'origine eta », a dans tous les cas préparé les futurs candidats au prolétariat des mines de charbon de l'ère Meiji.

30. CHŪō YŪWA JigYō KYŌKAI, 1974 [1939], p. 77-101.

31. Aso, 1979, p. 63-76. 


\section{De Meiji à Shōwa :}

\section{une présence accrue des burakumin dans l'industrie du charbon}

Le développement de l'extraction industrielle du charbon s'accélère durant l'ère Meiji, surtout à partir des années 1890 . À ce volume de production croissant, correspond une augmentation du nombre de travailleurs charbonniers, qui de 30345 individus en 1893, passe à 88330 en 1904, pour l'ensemble du Japon ${ }^{32}$.

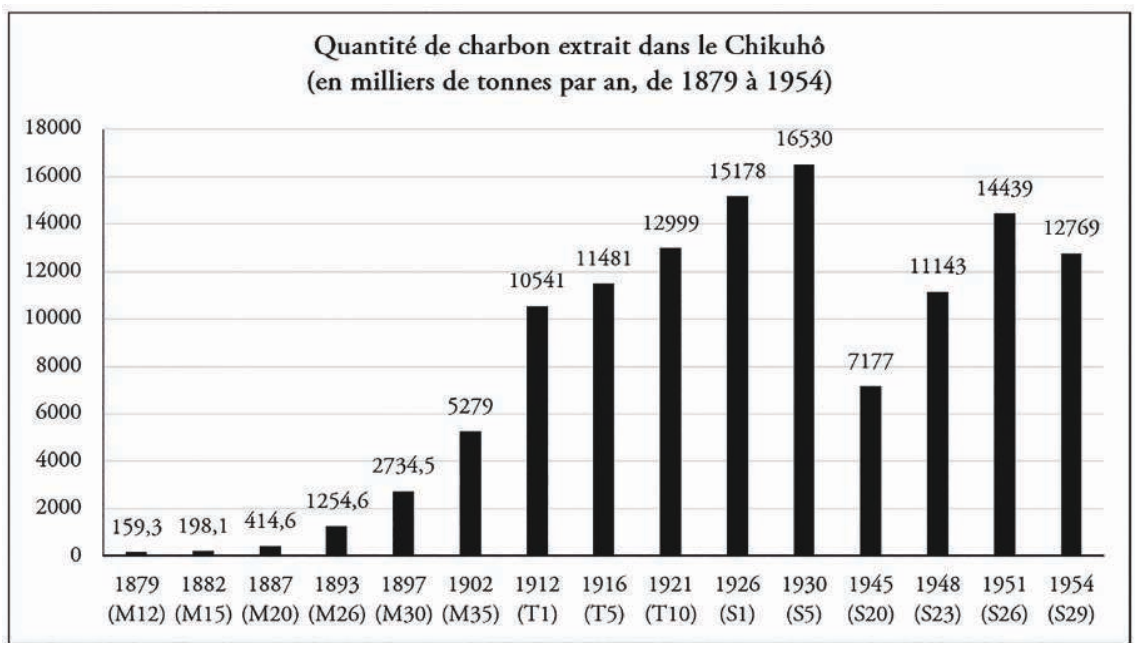

FIGURE 2. ÉVOLUTION DE LA PRODUCTION DE CHARBON DANS LE CHIKUHŌ DE 1879 À $1954^{33}$.

On pourrait supposer qu'avec la Révolution industrielle et l'essor du capitalisme, les burakumin puissent s'impliquer dans l'activité charbonnière selon d'autres modalités que celles de l'ancien régime - d'autant que ces burakumin, devenus 《 nouveaux citoyens 》(shin heimin 新平民) depuis l'ordonnance

32. Tsūshō SANGYōSHŌ, 1964, p. 194-195.

33. YADA, 1974, p. 34-75. 


\section{CIPANGO}

74 Cahiers d'études japonaises $n^{\circ} 23$

d'émancipation (kaihōrei 解放令) ${ }^{34}$ de 1871, ont désormais le droit de choisir leur métier et leur lieu d'habitat. Pour les principaux historiens s'intéressant à cette question, tels que Mahara Tetsuo ou Nagasue Toshio ${ }^{35}$, il y a dans le cas du Chikuhō un lien évident entre burakumin et houillères, d'où l'adage : « là où il $\mathrm{y}$ a des terrils, il y a des buraku. Là où il y a des buraku, il y a des terrils » (botayama no aru tokoro ni buraku ari, buraku no aru tokoro ni botayama ari ボ タ山のある所に部落あり、部落のある所にボタ山あり) ${ }^{36}$. Comme de nombreux hameaux buraku étaient installés depuis l'époque d'Edo à proximité de gisements de charbon, des houillères ont naturellement été creusées près de ces buraku. D'abord pour des raisons géologiques : Nagasue Toshio affirme que « les principales houillères ouvertes dans les années 20 de l'ère Meiji [1888-1908] se situent pour la plupart dans, ou près des buraku [... ${ }^{37} \gg$, car leur situation topographique (gorges, flancs de colline, bords de rivière, etc.) était souvent favorable à l'ouverture de mines. Mais aussi pour des raisons socio-économiques : les terrains des buraku coûtaient moins cher et rencontraient moins de résistance à l'acquisition foncière, de sorte que l'implantation de mines y était plus aisée que sur les terrains des autres agriculteurs ${ }^{38}$. L'historien Kawamukai Hidetake assure

34. Promulguée le 12 octobre 1871 (28 août de la $4^{\mathrm{e}}$ année de l'ère Meiji), cette ordonnance met officiellement fin aux distinctions de statut concernant les populations de parias de type eta, hinin, shuku, tōnai, etc. : « les appellations [statuts] telles que eta, hinin, etc. étant abolies, leurs rangs et métiers doivent désormais être à l'égal des autres citoyens » (eta hinin tō no shō haiserare sōrōjō jikon mibun shokugyō tomo heimin dōyō tarubeki koto 穢多非人等ノ称被廃候条自今身分職業共平民同様タルヘキ事). Sans autres mesures politiques concrètes de lutte contre la discrimination, la ségrégation perdure néanmoins. De plus, eta et hinin perdent les privilèges liés aux métiers qui leurs étaient réservés, tout en devenant imposables, ce qui conduit finalement à appauvrir et prolétariser un certain nombre d'entre eux.

35. Mahara, 1973, p. 67-86 ; Nagasue, 1979, p. 98-107.

36. On retrouve cette expression chez la plupart des chercheurs qui travaillent sur cette question, comme chez Nagasue, 1979, p. 104, ou TAKi.I, 1985, p. 205.

37. Nagasue, 1989, p. 83.

38. Nagasue, 1979 , p. $99 \& 105$. 
ainsi que pour la région du Chikuhō, « sur les quelques cinq cents terrils existant actuellement, $[. .$.$] environ trois cents sont situés à proximité de buraku { }^{39} \gg$.

Il convient également de prendre en compte les mouvements migratoires des burakumin venus travailler dans les houillères, surtout à partir des années 1890 , selon certains chercheurs. Ainsi, pour Aso, c'est à ce moment précis, et avec cette immigration-là, que l'industrie du charbon dans le Chikuhō serait devenue une « industrie buraku ${ }^{40}$ ». Pour Mahara Tetsuo, plusieurs témoignages de mineurs « de troisième ou quatrième génération » confirmeraient que ces populations migrèrent en masse vers le Chikuhō avec la construction de ces houillères industrielles, pour constituer la forme archétypale des buraku actuels ${ }^{41}$. Les enquêtes de Nagasue Toshio sur les transmissions orales des buraku semblent indiquer que cette immigration provient en premier lieu des régions du Chikuzen et du Buzen, extérieures au Chikuhō, ainsi que du Shikoku et du Chūgoku ${ }^{42}$. Pour Shindō Toyo.o 新藤東洋男 (1932-), «le nombre de buraku a augmenté de manière considérable, et proportionnelle au développement des houillères ${ }^{43}$. 》 Taki.i Yoshitaka 滝井義高, lui, se base sur l'exemple des habitants d'un buraku d'Ikeura, dans le district de Munakata (département de Fukuoka), qui en 1897, abandonnèrent conjointement leurs terres agricoles ancestrales pour s'installer tous ensemble dans le district de Kurate, afin d'y vivre du travail dans les mines ${ }^{44}$.

Les statistiques dont on peut disposer pour l'époque nous conduisent néanmoins à relativiser la proportion de burakumin parmi les populations venues travailler dans les houillères, du moins pour la période antérieure aux années 1920. Si le nombre de burakumin a triplé dans le district de Tagawa entre 1852 et 1920, passant de 3745 à 11243 individus, leur pourcentage au sein de la population totale du district a dans le même temps baissé de $12,5 \%$ à $7 \%$ (figure 1 et note

39. KaWAmukai, 1989, p. 115.

40. Aso, 1979 , p. 84 .

41. Mahara, 1973, p. 69.

42. Nagasue, 1989, p. 99.

43. ShIndō, 1978, p. 166.

44. TAKi.I, 1985, p. 206. 
correspondante). Quant au rythme de croissance démographique, il s'établit en moyenne à 7,7 \% par an dans le district, mais n'est que de 4,4\% parmi les burakumin, soit un taux équivalent à la moyenne nationale de croissance de la population buraku, alors de $4,3 \%$ par an ${ }^{45}$. Il reste cependant difficile de déterminer à quel moment cette hausse démographique aurait été particulièrement marquée : à notre connaissance, il n'existe pas pour cette période de recensement burakumin qui serait à la fois consultable publiquement ${ }^{46}$, et pratiquerait un découpage géographique constant. L'enquête publique de 1868, par exemple, se base sur les fiefs ( han 藩) d'Ancien Régime, et non sur les districts (gun 群).

La tendance générale laisse tout de même à penser que si immigration il y a eu, celle-ci concerne d'abord, en volume, les non burakumin, d'autant que les chiffres relatifs à la population buraku de l'époque sont plutôt fiables et exhaustifs. Pour les périodes plus récentes, les burakumin ne sont en effet comptabilisés comme tels que s'ils habitent dans un buraku reconnu par le gouvernement; tel fut par exemple le cas dans les enquêtes publiques conduites de 1970 à 1990. Dans les enquêtes qu'il diligenta durant la première moitié $\mathrm{du} \mathrm{xx}^{\mathrm{e}}$ siècle, le ministère de l'Intérieur devait en sus recenser le nombre de ceux qui n'habitaient pas dans les buraku, à partir du « lieu d'habitat des ancêtres » (honseki 本籍) enregistré dans leur état civil ${ }^{47}$.

À partir des années 1920, la situation semble évoluer. Si l'on considère le cas du district de Tagawa, l'immigration des burakumin vers les bassins houillers prend de l'ampleur : la proportion de burakumin au sein de la population générale passe de $7 \%$ en 1920, à $10 \%$ en 1933. En valeur absolue, le nombre de burakumin,

45. Taux calculé à partir des enquêtes menées par le ministère de l'Intérieur en 1871 et 1921 (Chūō yūWA JigYō KYŌKAI, 1974 [1936] n 40, p. 85). Ce fort taux est en partie dû au fait que l'enquête publique de 1933 sur les buraku avait été plus exhaustive que celle de 1920 . Il reste considérable comparé à celui du niveau national.

46. Des sources plus détaillées existent, mais elles restent dissimulées au sein d'archives privées.

47. Nous avons pu le constater en parcourant la reproduction de documents du début $\mathrm{XX}^{\mathrm{e}}$ siècle, comme les quatre-vingt numéros de Yüwa jigyō kenkyū (CHūō YūWA JIGYō KYŌKAI, 1974) et de Yūwa jigyō nenkan (CHŪō YŪWA JIGYō KYŌKAI, 1970), puis une dizaine de volumes de Kindai burakushi shiryō shüsei (1984-1987). 
qui s'élevait à 11243 individus en 1920, atteint 14727 en 1933 (figure 1). Une augmentation qui ne tient pas seulement de la natalité, car le taux de croissance annuel des burakumin est très élevé (10,1\% de moyenne annuelle pour le district de Tagawa, contre 8,6\% au niveau national durant la même période ${ }^{48}$ ).

Cette évolution se produit dans un contexte de diminution de la population générale du district, de 157407 habitants en 1920, à 146766 en $1933^{49}$. La période des années 1920 connaît en effet plusieurs crises successives, de l'après Première Guerre mondiale à celle de 1929, et l'on estime que le nombre de salariés dans les mines du Chikuhō aurait baissé de plus de $30 \%$ entre 1919 et 1926, puis de $50 \%$ entre 1926 et $1932^{50}$. Peut-on alors expliquer l'augmentation démographique des burakumin au cours de cette période d'agitation par le recours à des salariés à bas coût ? Le cas des immigrants coréens au Japon, dont les données sont plus aisément accessibles que pour les burakumin, nous laisse le supposer, puisque leur nombre augmente en effet constamment durant ces mêmes années 1920. De 30189 Coréens au Japon en 1920, on estime qu'ils étaient 171275 en $1927^{51}$, soit presque six fois plus en l'espace de sept années. Avec une population coréenne de 14595 individus, Fukuoka se hisse en 1927 au troisième rang des départements les plus peuplés par les Coréens, juste après Ōsaka (38 592) et Tōkyō $\left(\begin{array}{ll}15 & 030\end{array}\right)^{52}$. Nagasue Toshio estime que de nombreux émigrés de la péninsule ont été embauchés à la place des Japonais pour diminuer les coûts de main-d'œuvre durant ces années de crise. Trente houillères parmi les 97 que comptait le Chikuhō employaient ainsi un nombre considérable de Coréens en 1928, notamment des grandes entreprises telles que Mitsubishi,

48. Taux calculé à partir des enquêtes effectuées par le ministère de l'Intérieur en 1921 et en 1935 (Yüwa jigyō kenkyū, op. cit., n 40, p. 85 ; CHŪō YŪWA JIGYō KYŌKAI, 1970 [1935], p. 283-287).

49. Chikuhō Sekitan KōgYōshi nenpyō hensan inkai, 1973 ; SAKamoto, 1997, p. $84 \& 107$.

50. Yada, 2014., p. 67 ; NAGASUe, 1973, p. 133, \& 1989, p. 92.

51. TōKYō CHIHŌ SHOKUGYŌ SHŌKAI JIMUKYOKU 東京地方職業紹介事務局, 2000 [1928], p. 199.

52. Ibid., p. 197. 


\section{CIPANGO}

78 Cahiers d'études japonaises nº23

Furukawa, Kaijima, Asō, ou Kurauchi ${ }^{53}$. Il est toutefois possible que les crises des années 1920 aient aussi favorisé l'emploi conjoint de burakumin aux côtés des Coréens dans certains secteurs, dont les mines. Tel fut par exemple le cas dans les houillères d'Asō Takichi 麻生太吉 (1857-1933) ${ }^{54}$.

La majorité des chercheurs s'accorde à penser qu'un certain nombre de burakumin figuraient parmi les ouvriers des houillères du Chikuhō dès l'ère Meiji : pour Kawamukai Hidetake, il y avait « de nombreux burakumin parmi les mineurs de charbon » aux ères Meiji comme Taishō (1912-1926) ${ }^{55}$. Mahara Tetsuo va jusqu'à affirmer que « les burakumin constituent presque $60 \%$ des ouvriers dans les mines des grandes entreprises, voire $80 \%$ dans celles des PMI ${ }^{56}$. » Aso Tatsuo, lui, écrit qu' «à partir de la seconde moitié de l'ère Meiji, le lien entre buraku et charbon devient si important, que l'on peut considérer l'industrie houillère comme une industrie buraku » (buraku sangyō 部落産業) ${ }^{57}$. Généralement employé pour qualifier l'industrie du cuir, ce dernier terme de buraku sangyō désigne une spécialisation professionnelle et son fonctionnement : il sous-entend que ses travailleurs se situent au plus bas de l'échelle des valeurs et que ces communautés ont la responsabilité, voire le monopole du secteur.

On pourra se demander, devant le manque de sources disponibles pour l'ère Meiji ${ }^{58}$, si l'importance des burakumin dans l'univers du charbon n'a pas été exagérée, en particulier par les historiens spécialistes des buraku. La perception de l'époque plaide pourtant en faveur de l'omniprésence de cette population dans les houillères. Sano Manabu 佐野学 (1892-1953), l'un des leaders et théoriciens du Parti communiste japonais ${ }^{59}$, écrit ainsi en 1923 que « dans le département

53. Nagasue, 1989, p. 92.

54. SHINdō, 1978, p. 119.

55. KaWAMUKaI, 1989, p. 114.

56. Mahara Tetsuo, cité par SHINdō, 1978, p. 38.

57. Aso, 1979, p. 84.

58. Sakamoto, 1997, p. 82.

59. Sano fera cependant sa déclaration de « conversion politique » (tenkō 転向) en prison, l'année 1933. 
de Fukuoka, le peuple eta [eta-zoku エタ族] compte presque 70000 individus, dont la plupart sont mineurs ${ }^{60}$. » De même, Matsumoto Kichinosuke 松本吉 之助 (1902-? $)^{61}$, jadis mineur dans le district de Kaho, précise que « les femmes de [son] buraku travaillaient toutes à la houillère ${ }^{62}$. »Certains documents d'époque permettent de constater la récurrence de la profession minière parmi ces populations jusqu'aux alentours de Fukuoka, où l'industrie du charbon était pourtant moins développée que dans le Chikuhō. Le duc Tokugawa Iesato 徳川家達 (1863-1940), président de la délégation de l'association Saiseikai 済生会 ${ }^{63}$, rapporte ainsi sa visite en 1917 d'un buraku de Jigyōnishimachi 地行 西町, dans la localité de Fukuoka, le décrivant comme majoritairement constitué « de mineurs, terrassiers, ainsi que des colporteurs de légumes, poissons salés et autres produits séchés ${ }^{64}$. »

Si les mineurs seraient nombreux parmi les burakumin, ces derniers seraient, selon les perceptions, tout aussi nombreux parmi les mineurs. Matsumoto Kichinosuke affirme ainsi qu' « il devait y avoir un nombre considérable de burakumin parmi les ouvriers des mines de Chikuhō », et explique avoir assisté de nombreuses fois à des scènes où des mineurs buraku cachaient leur origine en insultant d'autres burakumin d'《eta-gorō » (エ夕 五郎), lorsqu'il travaillait lui-même dans des houillères, notamment celles en régie d'État (kan.ei 官営) de Chūō 中央 et d'Uruno 潤野 (Kaho), au cours des années $1920^{65}$. Citons également le témoignage de Shirato Zentarō 白土善太 郎, ingénieur de la compagnie minière Meiji Tankō, qui parmi ses souvenirs des années 1890-1900, note que « les mineurs du charbon étaient considérés par les agriculteurs des villages comme des habitants des hameaux spéciaux [tokushu

60. SANO, 1923.

61. Un des premiers membres de la Suiheisha de Kyūshū, proche du PCJ.

62. Matsumoto, 1977, p. 12.

63. Association d'aide sociale paragouvernementale fondée en 1911.

64. Saiseikai kaihōo 済生会会報 (Bulletin de l'association Saiseikai), n 4, 6 janvier 1918, reproduit dans Kindai burakushi shiryō shüsei, 1984-1987, vol. 9, p. 330.

65. Matsumoto, 1977, p. 31. 


\section{CIPANGO}

80 Cahiers d'études japonaises nº 23

buraku 特殊部落 $]^{66} \gg$, montrant à quel point d'amalgame les burakumin étaient perçus comme omniprésents parmi les ouvriers des mines.

Les données statistiques disponibles pour cette période suggèrent toutefois une réalité plus nuancée. Le graphique infra (figure 3), issu des chiffres de l'enquête publique de $1933^{67}$, révèle tout d'abord que les burakumin sont loin d'être majoritaires parmi les mineurs de Chikuhō. Même à Tagawa, où le taux de présence des burakumin parmi la population est particulièrement élevé (10\% des habitants du district en 1933), le nombre total d'individus burakumin, soit 14727 personnes, représente moins du tiers de la population minière de la zone (53216 mineurs).

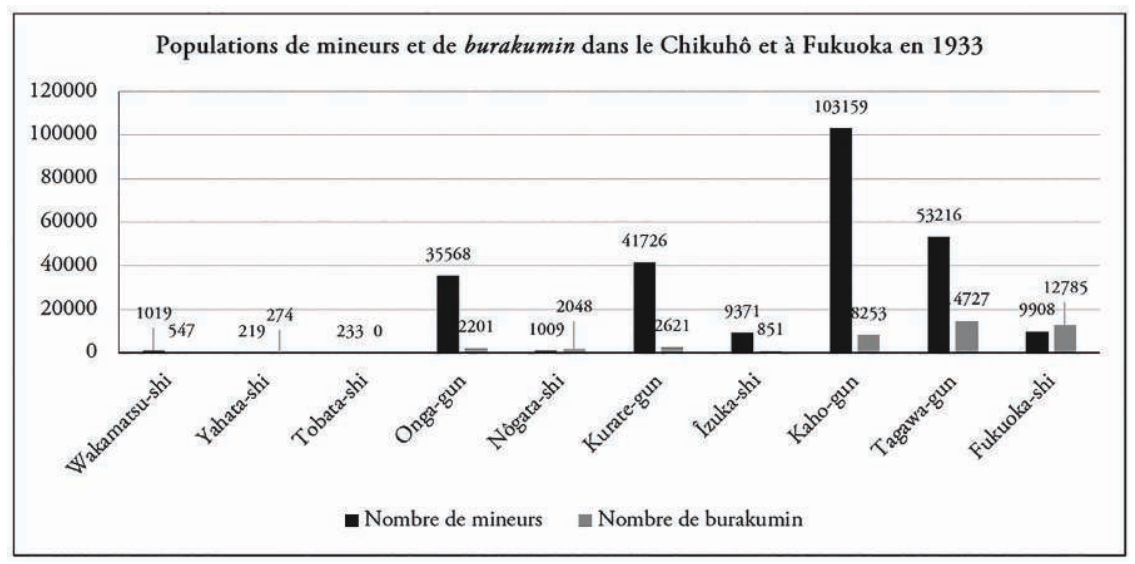

FIGURE 3. BURAKUMIN ET MINEURS DANS LE CHIKUHŌ ET DANS LA LOCALITÉ DE FUKUOKA EN $1933^{68}$.

66. Cité par Mahara, 1973, p. 81. Sur Shirato Zentarō, nous nous référons à NAKAMURA, 2008.

67. Sur le plan du nombre de salariés dans les mines, le niveau de l'année 1933 est globalement équivalent aux années précédant la prospérité provoquée par la Première Guerre mondiale. Chiffres de la population ouvrière cités par YADA, 2014, p. 67.

68. D'après SAKамото, 1997, p. 84. 
Il y a cependant une certaine corrélation entre la présence de mines et celle de buraku. Ainsi, dans les zones urbaines (celles des « localités », shi 市), la présence des burakumin comme des mineurs est faible : la ville de Wakamatsu ne compte par exemple que 547 burakumin et 1019 mineurs. De même dans les zones rurales (celles des « districts », gun) situées en aval de la rivière Onga, comme dans le district d'Onga, qui ne compte que 2201 burakumin pour 35568 mineurs. Les besoins en main-d'œuvre pour le transport du charbon sont en revanche plus importants en amont, sur le cours supérieur de l'Onga et ses affluents, dans ces zones montagneuses et reculées où se situent les sites d'extraction. On y retrouve par conséquent une présence accrue de mineurs, mais aussi de burakumin : 14727 burakumin et 53216 mineurs dans le district de Tagawa, 8253 burakumin et 103159 mineurs dans celui de Kaho. Là où il y a des mines, et donc des mineurs, la taille des communautés buraku se montre proportionnelle au besoin de main d'œuvre, du moins dans le Chikuhō. Les gérants des houillères ont donc certainement profité de la forte présence burakumin déjà sur place, ainsi que de l'immigration de ces populations.

Parmi les buraku établis à proximité des mines, certains hameaux dépendaient effectivement des activités liées au charbon. Ainsi, Tagawa et Kaho se révèlent être les deux districts où le plus fort nombre de buraku reposaient partiellement ou exclusivement sur le secteur houiller. 


\section{CIPANGO}

82 Cahiers d'études japonaises n ${ }^{\circ} 23$

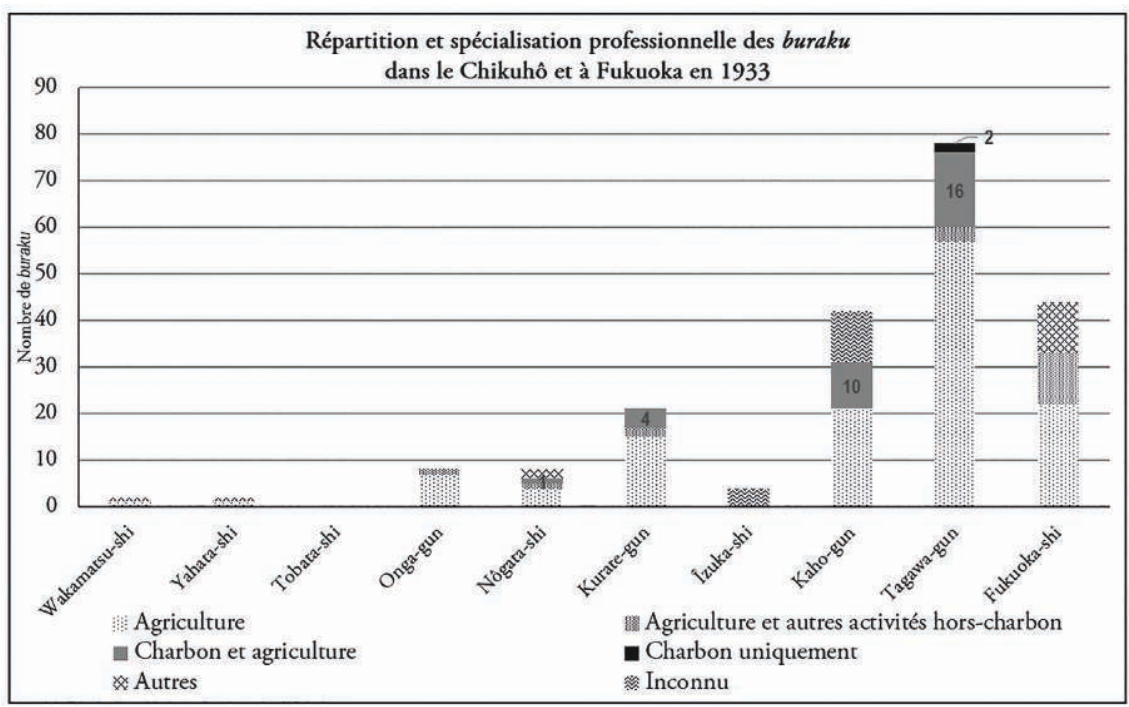

\section{FIGURE 4. SPÉCIALISATION PROFESSIONNELLE DES BURAKU DANS LE CHIKUHŌ ET À FUKUOKA EN $1933^{69}$.}

À Tagawa, deux buraku situés à l'intérieur même des mines vivent alors uniquement du travail dans les houillères : le buraku de la mine $\mathrm{n}^{\circ} 2$ d'Ōmine (village de Kawasaki, alors géré par l'entreprise Kurauchi Kōgyō 蔵内鉱業) et celui de la mine de Kigyōkomatsu 起行小松 (bourg de Gotōjimachi 後藤寺町, géré par le Kyūshū Kōgyō 九州鉱業, sous la direction d'Asō). Le premier compte 215 habitants, soit presque le tiers des 739 salariés de la houillère; le second est quant à lui plus petit, avec seulement 90 habitants, formant $14 \%$ des 630 salariés de la mine ${ }^{70}$.

Dans le district de Kaho, un tiers des buraku complète ses revenus agricoles par le travail minier, ce qui nous éclaire sur la place du charbon pour les buraku de cette partie du Japon : lorsque l'agriculture ne suffit pas à subvenir aux besoins de ces populations, c'est en effet l'industrie houillère qui leur assure un revenu

69. Ibid.

70. Ibid., p. 91 ; ТокітA, 1976, p. 75. 
complémentaire, et non la tannerie ou l'artisanat, comme souvent dans les buraku des autres régions. Ainsi, pour les quatre gun de Chikuhō (Tagawa, Onga, Kurate, et Kaho), trente buraku vivent à la fois de l'agriculture et du charbon, contre seulement six qui complètent leurs revenus agricoles par des activités non liées au charbon.

Ces burakumin agriculteurs - dont le taux élevé renvoie à la présence historique, au cours de l'époque d'Edo, de villages eta dans la région - ont pu jouer un rôle de « réservoir de main-d'œuvre minière » du fait de leur proximité géographique avec les mines, et de leur statut plus précaire que chez les autres agriculteurs. L'enquête nationale de 1931 montre ainsi que le taux de métayers chez les burakumin était alors le double de la moyenne nationale (52\% contre $27 \%)^{71}$, tandis que le besoin de main-d'œuvre dans les houillères continua à se faire croissant jusqu'à la fin des années $1940^{72}$. Si nous ne disposons pas de chiffres précis pour le Chikuhō, le pourcentage national d'agriculteurs burakumin allait toutefois diminuer en l'espace d'un quart de siècle (de $51 \%$ en 1931 à $46 \%$ en $1958^{73}$ ), reflétant certainement une mobilité vers le secteur industriel.

Le pourcentage de mineurs dans les buraku du Chikuhō semble avoir d'autant plus augmenté dans l'après-guerre que l'appel à la main-d'œuvre coréenne devenait moins évident. Ainsi, une enquête de 1950 menée dans le district de Kurate révèle que la proportion d'ouvriers du secteur minier y est plus élevée dans les buraku que dans les autres hameaux. Dans les communes comme Furutsuki, les quartiers buraku peuvent ainsi compter jusqu'à $94 \%$ de mineurs. Alors que les secteurs de Kasuga-Ouest et Kasuga-Est comptent en moyenne 64 \% de mineurs, les deux autres secteurs de la commune, marqués par une forte présence de mineurs mais sans présence buraku, présentent des pourcentages bien plus faibles : $46 \%$ de mineurs pour le quartier d'Ideguchi, et seulement $30 \%$ de mineurs à Kamikanzaki ${ }^{74}$.

71. Enquête publiée dans CHŪō YŪWA JIGYŌ KYŌKAI, 1970 [1933], p 556.

72. YADA, 2014, p. 67.

73. Chūō YŪWA JigYō KYŌKAI, 1970 [1933], p. 556 ; BURAKU KaIHō KENKYŨJo, 1981 , p. 55.

74. Enquête effectuée par Tsuru Daijirō 都留大治郎, citée par ToKITA, 1976, p. 72. 
Ce taux élevé de mineurs burakumin est confirmé par l'enquête nationale de 1958, selon laquelle 2,4\% d'entre eux travaillent dans l'industrie houillère. Un chiffre certes faible à l'échelle nationale, mais deux fois supérieur au pourcentage de mineurs parmi le reste de la population, qui n'est que de $1,1 \%{ }^{75}$. D'après une autre enquête publique effectuée en 1973 dans le district de Tagawa, $33 \%$ de la population buraku déclare avoir déjà travaillé dans une houillère ; parmi eux, $63 \%$ n'ont travaillé que dans des mines de $\mathrm{PME}^{76}$. Dans la mesure où ces mines employaient également de nombreux travailleurs non buraku, on peut se demander si l'industrialisation a eu pour effet de fondre les burakumin dans la masse indifférenciée du prolétariat ou si, au contraire, la ségrégation et la discrimination issues de l'ordre ancien se sont maintenues au sein des houillères.

\section{La condition burakumin dans les houillères industrielles du Chikuhō}

Au lendemain de l'ordonnance d'émancipation, des émeutes paysannes éclatent en signe de protestation contre l'accession à la citoyenneté ordinaire des eta et des hinin, principalement dans le Japon de l'ouest. Le département de Fukuoka est celui où les émeutes ont été, de loin, les plus importantes du Japon, avec 64000 participants arrêtés et sanctionnés (soit $35 \%$ de l'ensemble des émeutiers, estimé à 182000 au niveau national). Quant au nombre de foyers de buraku incendiés, les chiffres varient entre 550 et 2000 foyers au niveau départemental, pour un total de 1050 à 2500 foyers détruits à l'échelle du Japon ${ }^{77}$. Le soulèvement avait débuté chez les agriculteurs du Chikuhō, notamment ceux du district de Tagawa et de Kama (par la suite intégré à celui de Kaho), avant de s'étendre à l'ensemble du département. Là, les émeutiers avaient demandé à ce que « les eta restent comme avant des eta », entre autres revendications, telles que la baisse des impôts. S'il conviendrait de s'intéresser plus en détail aux motivations des émeutiers, ce soulèvement nous révèle en tout cas que la forte présence burakumin dans ces régions n'amoindrissait pas pour autant l'hostilité du reste de

75. Buraku Kaihō Kenkyūjo, 1981, p. 55.

76. Saкамото, 1997, p. 106.

77. Kindai burakushi shiryō shüsei, 1984-1987, vol. 2, p. 3 \& 584. 
la population à leur égard. On retrouve ainsi sans grande surprise tout un système de discrimination au sein des mines, que Shindō Toyo.o et Matsumoto Kichinosuke évoquent dans de nombreux récits ${ }^{78}$.

\section{La discrimination dans l'organisation du travail}

$\mathrm{Au}$ sein des houillères industrielles, l'aspect le plus direct et quotidien de la ségrégation est la présence de baraquements spéciaux où logent les burakumin (les «baraques d'eta », eta-naya エ夕納屋), ainsi que de bains à leur usage (les eta-buro エ夕風呂). Ceux-ci apparaissent à partir de la fin du XIX ${ }^{\mathrm{e}}$ siècle, et accompagnent le développement des houillères. Un rapport $\mathrm{du}$ « Conseil pour améliorer les situations des buraku pauvres » (Saimin buraku kaizen kyōgikai 細 民部落改善協議会), organe dépendant du ministère de l'Intérieur, note ainsi en 1912 que « certaines mines font habiter toute une race [ou tribu, shuzoku 種族] ensemble, dans un même espace délimité ${ }^{79}$. » On retrouve ces eta-naya et eta-buro dans les mines du groupe Mitsubishi à Namazuta 鯰田, dans celles d'Asō Takichi, comme à Mameda豆田 et Kamimio 上三緒, de même que dans les mines en régie d'État d'Uruno et de Chūōo ${ }^{80}$, laissant à penser qu'il s'agit d'une pratique répandue dans le Chikuhō, quel que soit le type de houillères.

Les eta-naya se distinguaient des autres baraquements d'ouvriers par leur construction particulièrement vétuste, avec des toilettes communes, sommaires et visibles de l'extérieur, car situées à l'extrémité de la baraque. Mahara Tetsuo se demande d'ailleurs si cette conception n'était pas destinée à signaler ouvertement les baraques de burakumin ${ }^{81}$. Quant aux eta-buro, ils sont en général plus petits et plus sales que les bains réservés aux autres ouvriers, et dans tous les cas communs et mixtes. Yamamoto Sakubē 山本作兵衛 (1892-1984), dont les dessins de mineurs du Chikuhō furent inscrits en 2011 au registre « Mémoire du monde »

78. Shindō, 1978, p. 185 ; Matsumoto, 1977, p. 31.

79. Rapport cité par SaKamoto, 1997, p. 103.

80. Matsumoto, 1977, p. 12 \& 103 ; SaKamoto, 1997, p. 91-102 ; Shindō, 1978, p. $13 \& 30-38$.

81. Mahara, 1973, p. 82. 
de l'Unesco, rapporte qu'à la mine de Kamimio, du groupe d'Asō Takichi, le bain des ouvriers buraku était nommé le «bain spécial 》(tokushu-buro 特殊 風呂), en écho au terme péjoratif de 《hameaux spéciaux 》 (tokushu buraku). Yamamoto précise qu'il était surtout le plus étroit (la moitié de la taille du bain des autres mineurs), et conclut que « la discrimination envers les gens des buraku continuait jusqu'aux bains. » Matsumoto Tsuya (1898 ?), salariée burakumin entrée à quatorze ans à la mine, témoigne de ce qu'elle vivait dans les années 1910 : «à l'époque, je ne savais pas pourquoi il existait des bains d'eta. Pour les gens ordinaires, il y avait deux grands bains. Et pour nous, c'était le plus petit et le plus sale. »

L'une des pires descriptions d'eta-buro concerne « le bain aux chevaux » de la mine de Namazuta, gérée par Mitsubishi. Selon Matsumoto Kichinosuke, les mineurs burakumin «s'y baignaient avec les chevaux. Leurs excréments flottaient dans les coins du bain. Je sentais alors jusqu'au fond de la moelle, et avec haine, ce que cela fait d'être discriminé. » Puis il ajoute : « ces choses-là ne sont pas marquées dans les archives. Moi-même, je n'ai pas voulu en parler jusqu'à présent ; j'en parle seulement maintenant, et pour la première fois ${ }^{82}$. $\gg$ D'après lui, ces eta-buro existaient dans de nombreuses houillères du Chikuhō ${ }^{83}$, même si peu de traces écrites attestent de l'existence d'un système de ségrégation fondé sur l'appartenance aux populations buraku.

Cette ségrégation, loin d'être circonscrite à l'espace de la vie quotidienne, se prolonge sans surprise jusqu'à l'organisation du travail. Non seulement les travaux attribués aux burakumin sont les plus pénibles physiquement, mais ils sont considérés comme subalternes, et seront les premiers à être remplacés par des machines de la fin de l'ère Meiji au début de l'ère Taishō, car pas assez rentables pour l'employeur. Ce fut notamment le cas pour le vidage de l'eau de l'intérieur des mines, opération devenue d'autant plus indispensable que les houillères de la fin de l'époque Edo avaient gagné en profondeur. Au tout début de l'ère Meiji, une majorité de burakumin occupait ces postes spécialisés dans

82. Témoignages de Yamamoto Sakubē, Matsumoto Tsuya et Matsumoto Kichinosuke cités par SHINDŌ, 1978, p. 31-32.

83. Matsumoto, 1977. p. 31. 
le vidage de l'eau. Ces ouvriers, dits mizukata (水方), disposaient de leurs propres quartiers dans les «baraques des videurs d'eau»(mizu-naya 水納 屋 $)^{84}$. Kaijima Tasuke 貝島太助 (1845-1916), qui fondera l'un des trois grands zaibatsu locaux ${ }^{85}$, explique ainsi qu'à l'époque où il n'était encore que « souschef » (tōryō 頭領), tous les videurs d'eau sous ses ordres étaient des burakumin. Avec l'introduction progressive, à partir de 1881, de machines à vapeur pour pomper l'eau, surtout dans les mines gérées par de grandes entreprises, ces ouvriers seront toutefois remplacés par des non-burakumin, plus qualifiés et compétents pour conduire les nouvelles machines.

Le travail de transport du charbon par charrette à bras (jinrikisha) ou embarcation (kawahirata) connaîtra le même destin. De la fin d'Edo au début de l'ère Meiji, les burakumin avaient été si nombreux à travailler dans ce secteur qu'un certain nombre de buraku composés d'immigrants s'étaient formés autour des mines. Ce fut par exemple le cas du grand buraku de Nakama 中間 (district d'Onga), qui ne comptait aucun village eta durant l'époque d'Edo. Avec les progrès accomplis à partir de 1891 par le chemin de fer, ces burakumin perdront eux aussi leur travail, notamment dans les grandes entreprises, pour être relégués vers les PMI moins mécanisées ${ }^{86}$.

L'emploi burakumin, d'autre part, se caractérise par des tâches à faible rémunération, critère souvent lié à la question de la qualification inférieure de ces populations. Ce phénomène se vérifie surtout dans les grandes entreprises, où les burakumin ont tendance à se voir attribuer les travaux les plus mal payés. De manière générale, on les retrouve plutôt à l'extérieur des houillères que dans le fond. Il a pu se dire dans les années 1890 et 1900 que la mine Mitsubishi de Nōgata 直方 ne permettait à aucun burakumin de travailler au fond de sa houillère ${ }^{87}$ : le bruit courut alors dans le Chikuhō qu'il ne fallait pas les laisser

84. Sakamoto, 1997, p. 100.

85. Kaijima est ainsi surnommé, avec Asō Takichi et Yasukawa Kei.ichirō, l'un des 《 trois Grands du Chikuhō » (Chikuhō gosanke 筑豊御三家).

86. Nagasue, 1989 , p. $80-86$.

87. Nagasue, 1979, p. 108. 


\section{CIPANGO}

toucher les mines, car ils les « souillaient ${ }^{88} \gg$. Mais il s'agissait surtout du secteur le mieux rémunéré, qui pouvait, par conséquent, faire l'objet d'une chasse gardée au sein de la société des mineurs. Selon l'enquête publique de 1927, la rémunération journalière moyenne du travail au fond des houillères est de 2437 yens, primes comprises, contre seulement 1309 yens pour le travail à l'extérieur des mines ${ }^{89}$. De fait, les travaux de fond, notamment l'extraction, sont rarement assignés aux burakumin, sauf durant certaines périodes exceptionnelles où la main d'œuvre vient à manquer, comme au début de l'ère Meiji (l'époque de jiyū-bori 自由掘り), ou pendant la guerre de Quinze ans (1931-1945) ${ }^{90}$.

À l'inverse, le saodori 棹取り, ou halage manuel des wagons à houille (hako ou tansha 炭車), est une tâche fréquemment attribuée à ces populations ${ }^{91}$. Elle est moins bien rémunérée lorsqu'elle est réalisée à l'extérieur de la mine : selon l'enquête de $1926 \mathrm{du}$ « Bureau de l'emploi d'Ōsaka »(Ōsaka chihō shokugyō shökai jimukyoku 大阪地方職業紹介事務局), relevant du ministère de l'Intérieur, le saodori pratiqué à l' intérieur des houillères est rémunéré en moyenne 1607 yens par jour, contre 1438 yens à l'extérieur ${ }^{92}$.

Les burakumin sont par ailleurs extrêmement nombreux au tri du charbon, un secteur plus féminisé, où les salaires sont encore plus faibles. D'après une autre enquête du ministère de l'Intérieur, datée cette fois de 1924, le salaire journalier moyen de ces postes est estimé à 1121 yens pour les hommes, et 892 yens pour les femmes, soit la moitié du salaire des mineurs de fond qui travaillent à l'extraction. La rémunération des tâches de fond se monte alors à 2051 yens pour les sakiyama 先山 directement chargés des opérations d'extraction, et à 2009 yens pour les

88. Mahara, 1973, p. 82 ; Shindō, 1978, p. 185.

89. TŌKYŌ CHIHŌ SHOKUGYō SHŌKAI JIMUKYOKU, Saitanfu rōdōjijō 採炭夫労働事 情, 1929, reproduit dans KYŪSHŪ DAIGAKU SEKITAN KENKYŪ SHIRYŌ SENTĀ, 2000, p. 190.

90. Sakamoto, 1997, p. 98.

91. Nagasue, 1979, p. 108 ; SaKamoto, 1997, p. 101 ; Mahara, 1973, p. 82.

92. ŌSAKA CHIHŌ SHOKUGYō SHŌKAI JIMUKYOKU, « Chikuhō tanzan rōdō jijō » 筑 豊炭山労働事情 (Situation du travail dans les mines du Chikuhō), enquête de 1926, reproduite dans Sekitan kenkyū shiryō sōsho, 1993, p. 81. 
atoyama 後山 qui traitaient le charbon une fois extrait, et qui bien souvent, étaient des femmes ${ }^{93}$.

Dans un texte de revendication du «Syndicat de l'industrie des houillères du Chikuhō » (Chikuhō sekitan kōgyō kumiai 筑豊石炭鉱業組合), voté en 1918 pour être envoyé au gouvernement, on peut lire : « les femmes qui travaillent au tri du charbon dans les mines de Chikuhō sont pour la plupart originaires des buraku spéciaux [... $]^{94}$. » Ueda Masayo, une ancienne trieuse burakumin, témoigne qu'à douze ans, en 1925, elle ne gagnait que 0,25 yens en travaillant quatorze heures par jour au sein de la mine Mitsui de Tagawa. Ce salaire particulièrement bas est dû à son jeune âge, au fait qu'elle soit une femme, mais certainement aussi à son appartenance au buraku. Ce dernier point explique qu'elle ne sache alors ni lire, ni écrire, et qu'elle est également moins productive. Comme elle l'explique : «mes amies trieuses connaissaient les lettres, mais moi, je comptais les wagons en déposant des pierres une à une ${ }^{95}$. $\gg$ De même, une ancienne femme-mineur rapporte ses souvenirs des années 1920 en ces termes : « il y avait une quarantaine ou une cinquantaine de jeunes filles qui venaient travailler au tri du charbon [...]. Elles étaient toutes différentes de nous, elles étaient des yottsu [...]. Il y avait aussi trois ou quatre filles normales qui venaient travailler au tri. Et elles restaient entre elles, de leur côté ${ }^{96}$. » Ce témoignage, en plus de rappeler l'usage du terme péjoratif de yottsu ${ }^{97}$, souligne que les mineurs ne se mélangeaient pas aux burakumin, même lorsqu'ils ou elles partageaient le même espace de travail.

Ajoutons également que le Tankōbushi, cette «Ballade des houillères » aujourd'hui si populaire dans l'ensemble du Japon, tire en grande partie son origine des refrains chantés par les ouvrières lors du tri du charbon. Si l'origine géographique de l'air du Tankōbushi fait l'objet d'une rivalité parmi les villes du

93. Naimushō, 2000 [1924], p. 25.

94. Cité par SaKamoto, 1997, p. 101.

95. UedA, 1977, p. 1014.

96. Témoignage cité par SHINDō, 1978, p. 34.

97. Le terme yottsu, « quatre », est souvent utilisé pour désigner les burakumin, insinuant qu'ils ne sont pas des êtres humains, mais simplement des êtres à « quatre membres ». 
Chikuhō, désireuses de s'en attribuer la paternité, ces chants étaient à l'époque attribués aux burakumin. Ueno Eishin 上野英信 (1923-1987), auteur non buraku de nombreux reportages sur les mineurs du Chikuhō, rapporte ce qu'une ancienne travailleuse de la mine lui avait raconté en 1947, alors qu'il était lui-même mineur dans la région : « tous appelaient le Tankōbushi "le chant des eta". Si jamais, ivres, on se laissait aller à le fredonner, on se faisait crier dessus [...]. Les Japonais ont leurs chansons japonaises, ce n'est pas la peine de chanter ce chant d'eta ${ }^{98}$. »Pour Harada Tomohiko, le Tankōbushi proviendrait du chant des trieuses burakumin de Tagawa, et probablement de la mine Mitsui ${ }^{99}$, tandis que Shindō Toyo.o situe plutôt son origine dans la mine Kaijima de Kurate ${ }^{100}$.

\section{La question de la relégation des burakumin vers le secteur des PMI-PME}

Si les houillères du Chikuhō entrent dans l'âge industriel et moderne durant l'ère Meiji, le capitalisme et la prolétarisation ne suffisent pas à effacer l'opprobre jeté sur les anciens eta et hinin, qui resteront des objets de mépris et de ségrégation de la part des autres ouvriers jusque dans les années 1920. Les archives du Suibei geppō ( «Bulletin mensuel de la Suiheisha de Kyūshū »), organe officiel de la Zen Kyūshū Suiheisha (ci-dessous ZKS) ${ }^{101}$, constituent un bon indicateur de la discrimination dans le Kyūshū des années 1920. Ce journal, publié mensuellement de juin 1924 à juin 1927, fait en effet paraître dans ses colonnes des lettres d'excuses de la part de personnes accusées d'actes discriminatoires anti-buraku par la Suiheisha. Pour ces dernières, ces lettres sont avant tout un moyen d'éviter les procès et pressions de la ZKS, dont les méthodes s'avèrent parfois musclées. Elles sont datées, signées et présentées d'une façon très

98. Cité dans Mahara, 1973, p. 76.

99. HaRADA, 1975 [1973], p. 231.

100. SHINDŌ, 1978, p. 180.

101. Formée en 1923, la Zen Kyūshū Suiheisha全九州水平社, ou Zenkyūsui, est une branche régionale de la «Société nationale des niveleurs », la Zenkoku Suiheisha, fondée l'année précédente pour lutter contre les discriminations envers les populations burakumin. 
formatée, reprenant les expressions typiques issues des textes de la Suiheisha, parfois quasiment à l'identique. Mais surtout, ces encarts indiquent le nom de l'auteur des excuses, son adresse, ainsi que le lieu où celui-ci a commis des actes jugés répréhensibles, ou prononcé de semblables paroles (document 1). Une soixantaine de ces lettres furent publiées durant les trois années de parution du journal, dont un tiers présentant leurs excuses à des mineurs du Chikuhō, ce qui confirme le fait que les houillères sont également des lieux de ségrégation envers les burakumin.

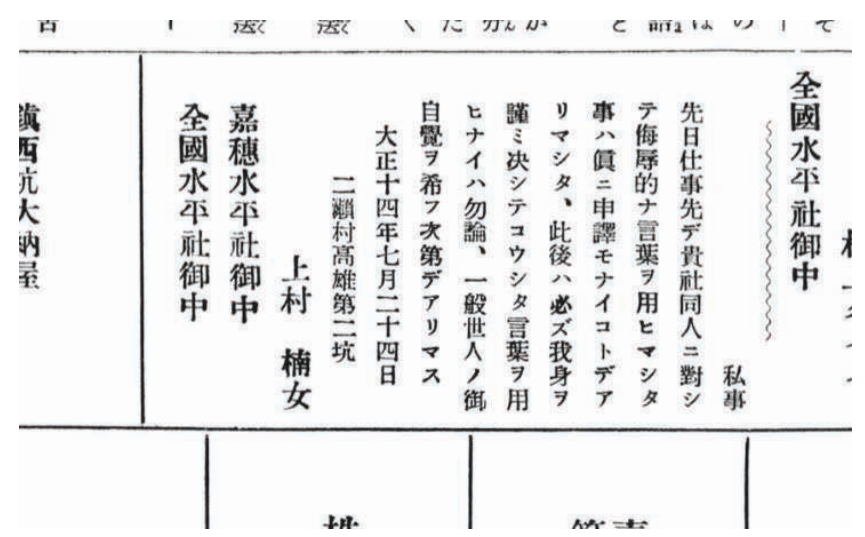

DOCUMENT 1. EXEMPLE DE LETTRE D'EXCUSE PUBLIÉE DANS LE SUIHEI GEPPŌ ${ }^{102}$.

Parallèlement à l'assignation chronique de tâches minières des moins rémunérées, les burakumin tendent aussi à être relégués vers le secteur des PMI-PME. Le phénomène se précise au fur et à mesure de l'industrialisation des mines. Mais fait-il vraiment système, et permet-il d'affirmer que les burakumin sont bien plus nombreux à travailler dans les PMI que dans les grandes entreprises privées, comme les zaibatsu ? C'est la position qu'adoptent

102. ZEN KyūShŪ Suiheisha, 1985, n 12 , le $1^{\text {er }}$ août 1925. 
92 Cahiers d'études japonaises $n^{\circ} 23$

Nagasue Toshio et Mahara Tetsuo ${ }^{103}$. L'idée paraît assez cohérente, même si les documents pour l'appuyer manquent, comme le souligne justement l'économiste Sakamoto Yūichi ${ }^{104}$.

L'étude des archives du Suihei geppō peut toutefois nous aider à y voir plus clair. Les lettres d'excuses mentionnées supra suggèrent en premier lieu la présence très probable de militants de la Suiheisha dans la mine, et par extension de burakumin, car ces lettres sont en général publiées à la demande de ces militants. D'autre part, les « cartes de visite » des bienfaiteurs du Suihei geppō constituent un second indicateur. Ces encarts publiés dans le mensuel comportent le nom, le titre ou l'appartenance, et l'adresse de l'annonceur (document 2), qui payait à cet effet des frais de parution selon l'espace occupé par son annonce. L'objectif de ces insertions varie naturellement entre un patron de restaurant et un homme politique, mais permet essentiellement aux responsables des mines d'exprimer leur volonté d'entente avec la Suiheisha, que ce soit en vue de nouveaux recrutements, ou pour s'attirer les bonnes grâces des burakumin travaillant déjà dans leur houillère. Si ces dirigeants prenaient la peine d'effectuer une telle démarche, on peut en déduire que les burakumin formaient alors une proportion non négligeable des salariés miniers. En ce sens, la nature et la quantité de ces cartes de visite peuvent être considérées comme des indicateurs relativement fiables de la présence buraku dans chaque mine.

103. Nagasue, 1989, p. 9092 ; Mahara, 1973, p. 82.

104. SaKamoto, 1997, p. 103. 


\begin{tabular}{|c|c|c|c|c|c|c|c|c|}
\hline $\begin{array}{l}\text { 日 } \\
\text { 高 } \\
\text { 日 } \\
\text { 出 } \\
\text { 東 }\end{array}$ & 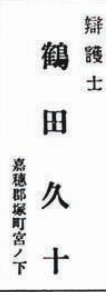 & 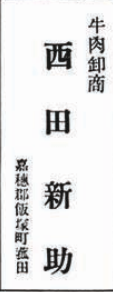 & 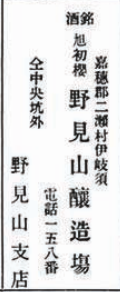 & 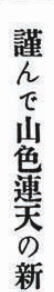 & 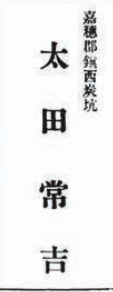 & 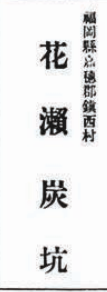 & 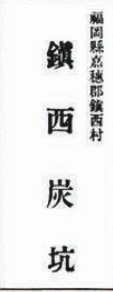 & $\begin{array}{l}\text { 芥 } \\
\text { 田 } \\
\text { 延 } \\
太 \\
\text { 太 } \\
\text { 郎 }\end{array}$ \\
\hline 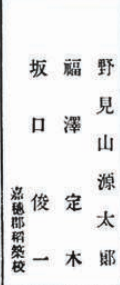 & 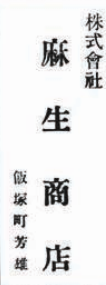 & 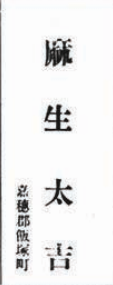 & 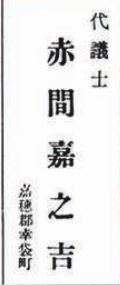 & $\begin{array}{l}\text { 年 } \\
\text { 通 } \\
\text { ! } \\
\text { ! }\end{array}$ & 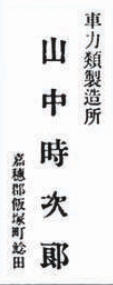 & 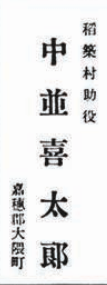 & 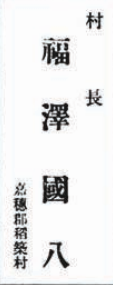 & 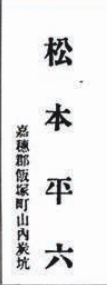 \\
\hline
\end{tabular}

DOCUMENT 2. EXEMPLE DE CARTES DE VISITE PUBLIÉES DANS LE SUIHEI GEPPŌ.

À noter, celle d'Asō Takichi ( $3^{\mathrm{e}}$ encart du bas, de gauche à droite), suivie de celle de son groupe $\left(2^{\mathrm{e}} \text { encart du bas, de gauche à droite }\right)^{105}$.

Il y a toutefois deux limites à l'exploitation de ces archives. L'une est temporelle : la période sur laquelle elles nous renseignent est de fait restreinte à celle de la publication de l'organe, entre juin 1924 et juin 1927. L'autre est d'ordre géographique : le Suibei geppō étant imprimé et distribué essentiellement dans le district de Kaho ${ }^{106}$, nos deux indicateurs s'appliquent surtout à des houillères de cette région. Ainsi les mines de Kaijima, essentiellement situées dans le district de Kurate, ne sont pas mentionnées dans les lettres d'excuses et les cartes de visite, alors que la présence d'ouvriers burakumin y est bien connue ${ }^{107}$.

105. Zen Kyūshū Suiheisha, 1985, nº 7, le $1^{\text {er }}$ janvier 1925.

106. HARAGUCHI, 1988, p. 495.

107. Sakamoto, 1997, p. 96, Shindō, 1978, p. 64. 


\section{CIPANGO}

94 Cahiers d'études japonaises $n^{\circ} 23$

Ces quelques précautions méthodologiques formulées, nous pouvons observer sur le plan quantitatif une corrélation entre la taille de la mine et le nombre d'indicateurs attestant d'une présence burakumin parmi ses ouvriers. Plus la mine est petite, plus le nombre de lettres d'excuses et de «cartes de visite » publiées est important : cinquante-trois occurrences en trois années pour l'ensemble des mines de moins de 1000 salariés, contre deux occurrences seulement pour toutes celles de 5000 à 6000 salariés (figure 5).

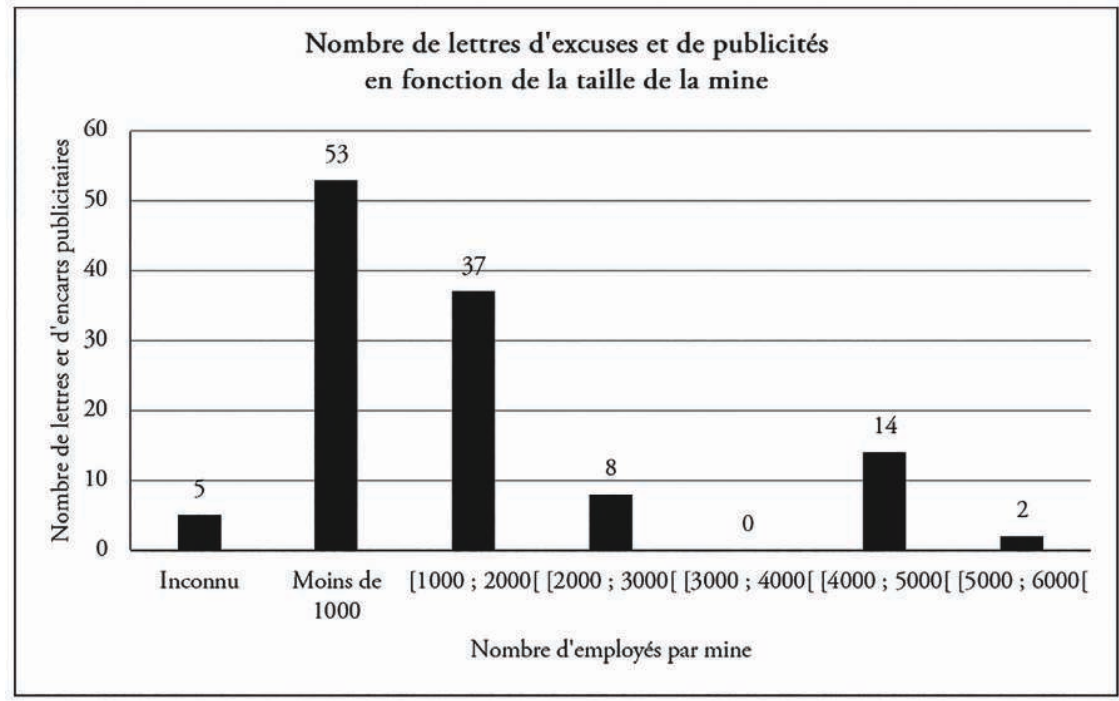

FIGURE 5. RÉPARTITION DU NOMBRE DE LETTRES D'EXCUSES

ET D'ENCARTS PUBLICITAIRES PARUS DANS LE SUIHEI GEPPŌ,

SELON LA TAILLE DE LA MINE CONCERNÉE ${ }^{108}$.

108. Zen Kyūshū Suineisha, 1985. Pour le nombre de salariés de chaque mine, nous nous référons à Moji tetsudō un’yuka門司鉄道局運輸課, Ensen tankō yōran 沿線炭鉱 要覽, 1928, cité par Sakamoto, 1997, p. 99. 
La seule exception à cette tendance concerne une mine en régie d'État, pour laquelle ces indicateurs révèlent une forte présence burakumin malgré un nombre d'ouvriers comparable aux effectifs des grandes entreprises privées. Il s'agit de la mine de Takao 高雄 (4 957 salariés), qui apparaît onze fois, contre deux pour la mine de Namazuta (Mitsubishi, 4548 salariés), et une seule pour celle de Tadakuma 忠隈 (Sumitomo, 4173 salariés). De manière globale, le nombre de lettres d'excuses et de cartes de visite relatives aux mines Mitsubishi et Sumitomo est clairement moindre (cinq en tout), laissant supposer un faible nombre de burakumin parmi leurs salariés. Une différence de stratégie entre les mines des grands groupes privés et celles en régie d'État peut néanmoins être décelée à partir de ces indicateurs : les mines gérées par les entreprises Sumitomo (Tadakuma), Mitsubishi (Namazuta) et Furukawa (Shimo-yamada 下山田) n'apparaissent en effet dans le Suihei geppō qu'à travers ces cinq lettres d'excuses, sans aucune publicité. Est-ce parce que les grands groupes privés ont tendance à préférer employer les non-burakumin, et à reléguer les burakumin à certains secteurs tels que le tri du charbon ou le transport, comme le supposent certains chercheurs ${ }^{109}$ ?

109. Nagasue, 1979, p. 108 ; Mahara, 1973, p. 82. 


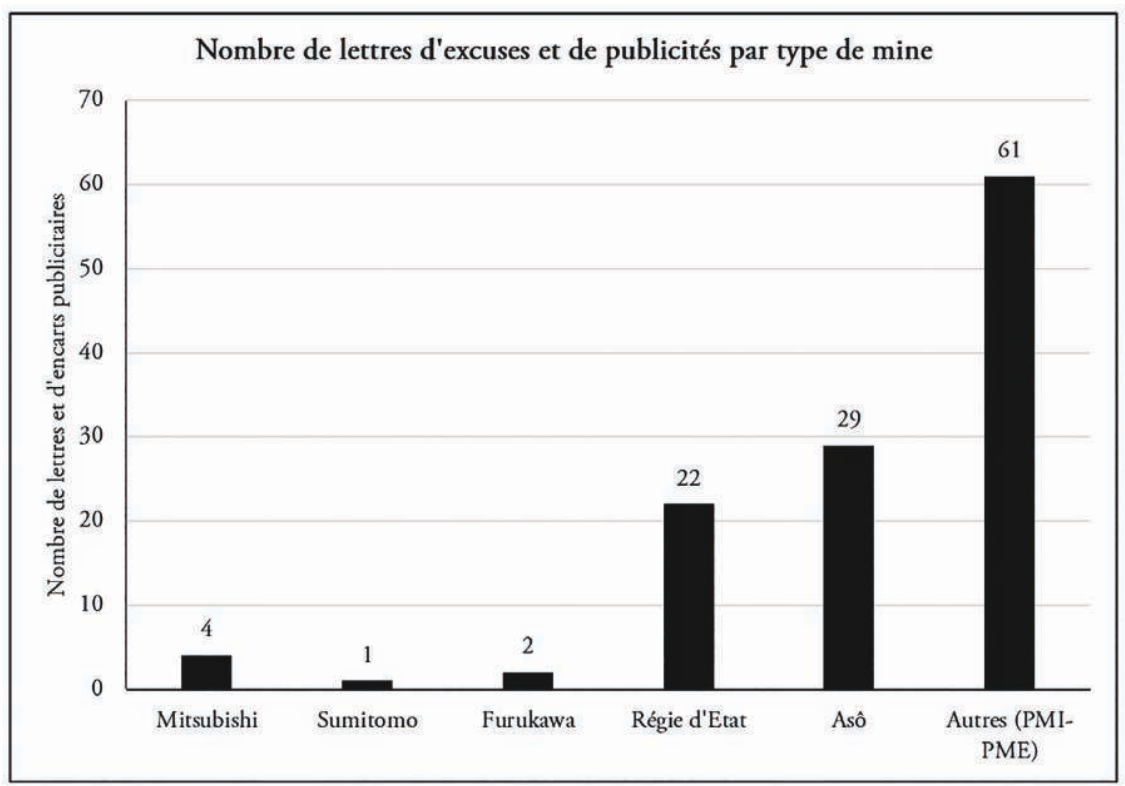

FIGURE 6. RÉPARTITION DU NOMBRE DE LETTRES D'EXCUSES ET D'ENCARTS PUBLICITAIRES PARUS DANS LE SUIHEI GEPPŌ,

\section{PAR TYPE DE MINE ${ }^{110}$.}

Alerté par les émeutes du riz de 1918 et le développement des mouvements ouvriers, Mitsui mena dans ses mines une politique de recrutement portant une attention particulière à l'état civil et aux antécédents des candidats, avec une « liste noire $\gg$ de militants des mouvements ouvriers ${ }^{111}$ où figuraient immanquablement les membres de la Suiheisha, alors très proches du PCJ. Par crainte des émeutes, certains de ces grands groupes choisirent d'autre part une politique d'isolement de leurs salariés. Ainsi, elles n'embauchèrent pas de burakumin dans les houillères situées à côté de leur buraku de résidence, ou ne les embauchèrent qu'à la condition

110. ZEN Kyūshū Suiheisha, 1985.

111. KANEKO, 1975, p. 100. 
qu'ils déménagent plus loin et ne voient plus leur famille ${ }^{112}$. Voici probablement pourquoi la mine Mitsui de Yamano (district de Kaho), qui compte pourtant de nombreux burakumin sur place, ne figure pas une seule fois dans le Suibei geppō.

Ajoutons qu’à partir des années 1930, les houillères appartenant à de grandes entreprises se mécanisèrent non seulement de plus en plus, mais accordèrent aussi davantage d'importance à la qualification. Ainsi, en 1931, $75 \%$ des mineurs de la mine $\mathrm{n}^{\circ} 3$ de Tagawa (Mitsui) étaient au moins diplômés de l'école primaire (jinjō shōgakkō 尋常小学校), et $31 \%$ d'entre eux possédaient un niveau d'éducation supérieur au collège (kōtō shōgakkō 高等小学校 $)^{113}$. Les burakumin, dont la qualification moyenne était inférieure à celle du reste de la population ${ }^{114}$, présentaient donc d'emblée moins de chances d'être embauchés dans ces houillères.

Quant aux mines en régie d'État affiliées à l'industrie sidérurgique de Yahata (Yahata Seitetsusho 八幡製鐵所), elles sont relativement bien représentées dans le Suihei geppō, en particulier celles de Takao, Chūō et Urushio 漆生. On les retrouve dans des lettres d'excuses, et surtout, à la différence des grands groupes privés, dans des « cartes de visite publicitaires » émanant de personnalités parfois relativement importantes. Ainsi, quatre chefs d'ōnaya 大納屋 ${ }^{115}$ de la mine de Takao formulent leurs vœux de nouvel an au Suihei geppō et à ses lecteurs par le biais de ces encarts publicitaires ${ }^{116}$. Ces insertions dans le journal laissent supposer que ces chefs d'ōnaya comptent un certain nombre de mineurs buraku sous leur responsabilité, mais qu'ils ont aussi intérêt à exprimer leur amitié pour les retenir chez eux et en attirer de nouveaux, puisque les recrutements relèvent effectivement de la fonction du chef de baraque, le naya-gashira 納屋頭 ${ }^{117}$. Dans un autre numéro, figurent deux cartes de visite de membres du conseil du bourg de

112. Nagasue, 1989, p. 90-91.

113. Sakamoto, 1997, p. 103.

114. ChŪō YŪWA JIGYŌ KYŌKaI, 1970 [1936], vol. 11, p. 135-136.

115. Grandes baraques des mineurs célibataires. NaGasue, 1979, p. 106.

116. Zen Kyūshū Suineisha, 1985, nº 7, le $1^{\text {er janvier } 1925 .}$

117. Pour l'organisation du système appelé « naya seido », nous nous référons à SumiYA, 2003 [1968], p. 323-329; Ogino, 1993, p. 41-43 \& 134. 
Kōbukuro 幸袋 appartenant également à la mine de Takao, dont un élu local ayant payé un encart particulièrement important ${ }^{118}$. Le but est électoraliste : en affichant son appartenance à la mine de Takao, il compte recueillir le suffrage des ouvriers buraku de cette houillère, ce qui sous-entend qu'ils doivent là encore représenter un nombre suffisamment important de voix pour justifier cet investissement.

En réalité, si ces mines en régie d'État ont mené une certaine politique d'embauche des burakumin, ce fut dans le contexte de la politique nationale de 《 réconciliation » (yūwa 融和) ${ }^{119}$ lancée suite aux émeutes du riz de 1918, qui avaient été attribuées à ces populations. Il s'agissait de leur fournir des aides afin de mieux les assimiler et les détourner des mouvements d'opposition, notamment des communistes.

Dans ces houillères, certains burakumin ont pu être employés dans des secteurs relativement « nobles » comme l'extraction du charbon. On peut relever au moins deux cas en 1918 et en 1921 pour les mines en régie d'État, alors qu'il faudra attendre les années 1930 pour observer des mineurs de fond burakumin dans les grands groupes privés ${ }^{120}$. Des cas de chefs de baraque burakumin sont par ailleurs avérés, comme ceux de la famille Wada (trois de 1899 à 1929 dans la mine d'Uruno, et un en 1931, dans la mine de Chūō) ${ }^{121}$. Ces naya-gashira, sous les ordres desquels travaillaient des mineurs de même origine, appartenaient à des familles plus riches de burakumin, et sont parfois même devenus des élus locaux. Leurs encarts publicitaires dans le Suibei geppō leur permettaient d'affirmer leur statut et de consolider leurs liens avec la communauté burakumin des mines, ou lors des élections ${ }^{122}$. Ainsi, contrairement aux groupes privés, les mines en régie d'État n'évitent pas ces populations habitant sur place. Elles privilégient plutôt la stabilité offerte par la main-d'œuvre locale en s'alliant aux notables buraku, qui en tant que chefs de baraques, peuvent garantir la paix sociale.

118. Zen Kyūshū Suiheisha, 1985, no 9 [sic: $\left.\mathrm{n}^{\circ} 10\right], 1^{\mathrm{er}}$ mai 1925.

119. Ancêtre de la politique d' «assimilation » (dōwa) mise en place par la loi de 1969. 120. D'après Saкамото Yūichi, l'un des chercheurs les plus documentés sur la question. 1997, p. 95-102.

121. Мatsumoto, 1977, p. $12-37 \& 113$.

122. Zen Kyūshū Suiheisha, 1985, nº 3, 8, 10, 15, etc. 
Disposant de nombreux métayers buraku dans le district de Kaho, Asō Takichi est d'abord un grand propriétaire terrien, profondément enraciné dans le Nord du Kyūshū. Avec un capital plus limité que les grands zaibatsu, il s'est très tôt intéressé à l'utilité spécifique de ces minorités discriminées pour les houillères de son groupe, l'Asō Shōten 麻生商店. En novembre 1888, il donne ainsi à un responsable de sa mine de Namazuta cette recommandation écrite : « il faudrait, avant que les autres mines ne s'en aperçoivent, recruter des mineurs parmi les nouveaux citoyens [c'est-à-dire les burakumin] ${ }^{123}$. » Une politique de recrutement qui conduira certains, tels Shindō Toyo.o, à décrire les mines d'Asō comme « celles où les burakumin du Chikuhō sont les plus nombreux à travailler ${ }^{124} \gg$.

Cette stratégie s'observe également dans la façon dont les mines de l'Asō Shōten insèrent des publicités dans le Suihei geppō. Quantitativement, les indicateurs de la présence burakumin dans une mine donnée font des mines d'Asō parmi les plus représentées dans les pages du journal, toutes lettres d'excuses et publicités confondues : vingt-neuf occurrences en trois ans, sans compter les publicités à titre individuel d'Asō Takichi lui-même (voir infra, document 2), qui achète à quatre reprises des encarts particulièrement imposants pour sa carte de visite. En réalité, Asō et ses mines sont présents dans pratiquement tous les numéros du Suibei geppō, devant les mines en régie d'État (vingt-quatre fois), et juste derrière celle de Chinzei 鎮西 (trente occurrences). Si cette dernière occupe le premier rang, sa particularité est d'être une PMI (de 518 ouvriers en 1928), gérée par des sympathisants et membres de la Suiheisha, et dont les salariés sont majoritairement burakumin ${ }^{125}$.

Comme les mines de Mitsubishi, ou celles à divers degrés en régie d'État, les mines d'Asō présentent des lettres d'excuses attestant de l'existence, au sein de ses houillères, de réelles tensions entre les burakumin et les autres ouvriers. Pourtant, le groupe Asō Shōten s'emploie dans le même temps à manifester sa bienveillance envers ces populations. En témoignent les publicités qu'il insère dans le Suibei geppō, qui ne proviennent pas seulement de ses chefs de baraques, mais également,

123. Directive d'Asō Takichi du 6 novembre 1888, citée par SAкамото, 1997, p. 100. 124. SHINDŌ, 1978, p. $124 \& 250$.

125. SAKAмотO, 1997, p. 100-103. 


\section{CIPANGO \\ 100 Cahiers d'études japonaises n²3}

et ce dès les premiers numéros, de responsables du plus haut niveau, tels que les directeurs des mines de Kamimio et de Sannai 山内. À cela s'ajoutent les publicités au nom de toute l'entreprise, sans compter celles de membres de la famille Asō, d'élus locaux ou d'industriels. Quantitativement, la famille Asō est aussi présente dans les pages du journal que la famille Wada, ces notables buraku dont certains membres sont chefs de baraques au sein de mines d'État, ou gestionnaires de petites houillères, comme celles de Chinzei et de Hanase 花瀬.

Ces publicités auprès des burakumin étaient d'autant plus nécessaires que les mines d'Asō sont « connues dans le Chikuhō pour la faible rémunération de leurs salariés et la longueur des journées de travail », d'après Yamamoto Sakubē, qui a lui-même travaillé chez Asō ${ }^{126}$. Un rapport rédigé en août 1932 par des ouvriers en lutte dénonce de même ces houillères, dont « les salaires sont $20 \%$ moins élevés que dans les autres mines » et où « les soins médicaux apportés aux ouvriers lors d'un accident du travail sont interrompus à la seule appréciation des contremaîtres, sans avis médical ${ }^{127}$. » Les auteurs de ce rapport sont majoritairement des travailleurs coréens, envers qui les burakumin du Chikuhō se sont fréquemment montrés solidaires. De nombreux Coréens, 1100 au début de l'ère Shōwa (1926-1989), travaillaient en effet dans les mines d'Asōo ${ }^{128}$ : leur cas, ajouté à celui des burakumin, révèle ainsi une stratégie d'utilisation préférentielle des groupes discriminés pour bénéficier d'une main-d'œuvre moins coûteuse.

\section{La question buraku au sein du mouvement ouvrier des mines du Chikuhō : alliance, ou subordination?}

Les mouvements politiques et syndicaux du Chikuhō n'échappent pas à la question buraku, surtout avec la création de la Zen Kyūshū Suiheisha en mai 1923. Du fait de l'importance démographique et de la force de cohésion des burakumin, cette organisation va jouer un rôle essentiel dans la structuration d'un

126. Yamamoto Sakubē, cité par SHINdō, 1978, p. 124.

127. Rapport des grévistes, ibid., p.126.

128. Ibid., p.124. 
mouvement social naviguant entre des logiques d'intérêt propre, d'une part, et de cet intérêt universel qu'appellent le marxisme et la lutte contre toutes les formes d'oppression. Au risque, parfois, de reléguer au second plan la question des discriminations spécifiques aux burakumin.

Les burakumin du Chikuhō ont joué un rôle aussi important que ceux de la ville de Fukuoka au moment de la fondation de la ZKS : Shindō Toyo.o affirme même que « la matrice de la Zen Kyūshū Suiheisha est née dans le Chikuhōo ${ }^{129}$. » Haraguchi Eiyū, spécialiste de la ZKS, situe ses prémices dans le district de Kaho ${ }^{130}$, ce que corrobore Matsumoto Kichinosuke ${ }^{131}$, alors employé à la mine d'Uruno, qui précise que la moitié des protagonistes à l'origine de cette initiative étaient des mineurs. Le siège de la Zenkyūsui se situant dans la ville de Fukuoka, chez Matsumoto Ji.ichirō 松本治一郎 (1887-1966), chef du comité exécutif, la région du Chikuhō et ses mineurs burakumin sont souvent associés aux actions de la ZKS. Quand il évoque les burakumin de Fukuoka ou de Kyūshū, Sano Manabu pense surtout aux mineurs de Chikuhōo ${ }^{132}$. De même, Kondō Hikaru 近藤光, un des acteurs de la création de la Suiheisha du Japon, croyait que le siège central de l'organisation se trouvait dans le district de Kaho, sis chez Hanayama Kiyoshi 花山清 (1896-1982)133, dont le buraku était alors majoritairement composé d'ouvriers de la mine de Chūōó ${ }^{134}$. Mais le village de Hanayama Kiyoshi est effectivement l'un des centres de la ZKS et le lieu où le journal Suibei geppō est hébergé, rédigé et imprimé 135 .

L'influence de la ZKS est importante parmi les mineurs buraku de Chikuhō, d'autant que le taux d'adhésion à la Suiheisha pour le département de Fukuoka est alors élevé ( $27 \%$, soit cinq fois la moyenne nationale, qui n'est que de $6 \%$ tout au

129. Ibid., p. 209.

130. HaRAgUCHI, 2001, p. 590.

131. Matsumoto, 1977, p. 39.

132. Sano, 1923.

133. Propos de Kondō, recueillis par la police lors de son arrestation de mai 1923. SHINDŌ, 1978, p. 209.

134. Ibid., p. 220.

135. Haraguchi, 1988 p. 495. 


\section{CIPANGO}

102 Cahiers d'études japonaises $\mathrm{n}^{\circ} 23$

plus $\left.{ }^{136}\right)$. Comme la Suiheisha au niveau national, la ZKS s'engage à défendre les burakumin en réclamant notamment aux individus ou institutions responsables de discrimination envers ces populations des excuses publiques, qu'elle reproduit dans le Suihei geppō. On lui doit aussi des actions concrètes en faveur de l'amélioration des conditions de vue des mineurs burakumin, comme l'abolition des eta-naya et des eta-buro, obtenue au cours des années $1920^{137}$.

\section{L'unité dans les luttes sociales et la grande coalition}

Entre lutte de classes et défense des intérêts particuliers des burakumin, la ZKS est confrontée aux mêmes dilemmes que la Suiheisha nationale quant à la position qu'elle devrait adopter envers les mineurs non burakumin. Elle opte néanmoins plutôt pour la solidarité de classe, au point de heurter, lors du troisième Congrès de laZenkoku Suiheisha (1924), certains membres du bureau central dont elle critique l'exclusivisme du mouvement, proposant de déployer davantage d'efforts pour obtenir la compréhension des non burakumin ${ }^{138}$. Sur le terrain, la ZKS est partie prenante des luttes pour la cause commune du prolétariat. La même année 1924, elle participe activement aux mouvements des travailleurs de la mine Mitsui de Miike 三池 ${ }^{139}$, mais aussi aux grèves de l'usine textile Harada Seimenjo 原田製綿 所 en $1925^{140}$, ainsi qu'aux mouvements des métayers (kosaku soggi 小作争議) du

136. En 1929, 18899 individus étaient membres de la Suiheisha parmi les quelque 70000 burakumin que comptait le département de Fukuoka (69 345 individus en 1920, 71913 en 1935). Au niveau national, la Suiheisha regroupait la même année 48483 membres sur le presque million de burakumin présents au Japon (829 674 recensés en 1921, puis 999687 en 1935). Sources : ChŪō YŪWA JiGYō KYōKAI, 1974, nº 40, p. 85 ; СHŪō YŪWA JIGYō KYōKAI, 1970 [1935], p. 283-287 ; Kindai burakushi shiryō shüsei, 1984-1987, vol. 9, p. 19 \& 29; HARAGUCHI, 1988, p. 502-503.

137. Témoignage de Yamamoto Sakubē, cité par SHIndō, 1978, p. 32.

138. Propos tenus au congrès de 1924 par Matsumoto Ji.ichirō et Hanayama Kiyoshi (archives personnelles).

139. SHindō, 1978, p. 230.

140. Certes, la moitié des grévistes étaient des femmes buraku. Haraguchi, 1989, p. 159. 
Chikuhō soutenus par le «Syndicat des paysans du Japon » (Nihon nōmin kumiai 日本農民組合), de 1923 à $1924^{141}$.

Certains buraku où la ZKS est puissante accueillent même les bureaux des grévistes non burakumin syndiqués afin de les protéger des intimidations musclées de leurs employeurs ou des chefs de baraque, lorsque ceux-ci font appel à la pègre locale. C'est le cas du buraku de Futase 二瀬 (district de Kaho), qui abritait le bureau du «Syndicat des mineurs de l'Ouest 》 (Seibu tankōfu kumiai 西部炭坑 夫組合) de la mine de Chūō, fondé en 1922. Les violences contre ces syndicalistes sont réelles : entre 1924 et 1925 , quatre grévistes sont grièvement blessés, et l'un meurt poignardé. Sa dépouille est déposée dans la tombe d'un burakumin ${ }^{142}$. Plus tard, en 1932, lorsque les grévistes du «Syndicat des mineurs de charbon du Japon » (Nihon sekitan kōfu kumiai 日本石炭坑夫組合) ${ }^{143}$ luttent pour l'amélioration des conditions de travail dans les houillères d'Asō, les buraku du district de Kaho sont mobilisés par la ZKS afin de leur fournir des denrées alimentaires, alors qu'ils en manquent eux-mêmes ${ }^{144}$.

À l'inverse, on observe également des exemples de solidarité de la part d'ouvriers non burakumin envers leurs confrères discriminés. Ainsi, en 1923, lorsque la Suiheisha mène campagne contre le maire du village de Nakamura (district de Kurate), accusé de discrimination anti-buraku, des travailleurs de la mine Kaijima d'Ōnoura 大之浦et des agriculteurs viennent leur prêter main-forte. Ensemble, ils obtiennent la publication par le maire d'une lettre d'excuses ${ }^{145}$.

Les militants de la Zenkyūsui n'ignorent pas, pour autant, que l' hostilité contre les burakumin n'est pas seulement le fait du camp capitaliste, mais qu'elle émane aussi de leurs propres camarades; ils constatent même que « la plupart des affaires de discrimination anti-buraku du moment se produisent au sein du prolétariat

141. SHINDō, 1978, p. 221.

142. Celle de la famille de Hanayama Kiyoshi, un des responsables du journal Suihei geppō.

143. Syndicat social-démocrate, comptant parmi les grévistes de 1932 de nombreux Coréens.

144. SHINdō, 1978, p. 111-126 \& 225.

145. Zen Kyūshū Suiheisha, 1985 [1924], articles des nº 3, 4 et 6. 


\section{CIPANGO \\ 104 Cahiers d'études japonaises $n^{\circ} 23$}

[musan kaikȳu 無産階級] ${ }^{146}$. » La ZKS tente alors de sensibiliser les camarades non burakumin afin qu'ils prennent conscience de leur appartenance à une même classe - celle des prolétaires -, et du non-sens que représente la ségrégation. En avril 1926, les femmes buraku lancent ainsi cet appel dans le Suibei geppō : « sœur non burakumin, ne nous discrimine pas, ne nous humilie pas, nous, les victimes du système de classe. Ce serait faire souffrir tes propres sœurs qui sont dans la même situation que toi, et finalement, te faire souffrir toi-même ${ }^{147}$. »

Le mot d'ordre que la Zenkyūsui tente de faire passer est alors celui de la 《 grande coalition pour l'intérêt commun » (daidō danketsu 大同団結) $)^{148}$, contre l'ennemi capitaliste. Dans cette confrontation, le racisme ${ }^{149}$ anti-buraku divise les burakumin des autres exploités, et « empêche considérablement la progression du mouvement prolétaire ${ }^{150}$. » Pour les militants de la ZKS, le combat contre le racisme et la lutte de classes constituent donc deux pôles fondamentaux et indissociables, l'un ne pouvant s'accomplir sans l'autre.

\section{La subordination à la lutte de classes}

L'union est également prônée au sein de la gauche, mais dans un sens plus spécifique : pour la gauche, la lutte d'émancipation des burakumin devrait se soumettre à celle, universelle, du prolétariat. Dans Seibu sensen 西部戦 線 («Le Front de l'ouest »), une revue proche du parti communiste publiée

146. Zen Kyūshū Suiheisha, 1985, nº 15, le 10 novembre 1925.

147. Ibid., n ${ }^{\circ} 18$, le $1^{\text {er }}$ avril 1926.

148. Ibid., n ${ }^{\circ} 12$, le $1^{\text {er }}$ août 1925.

149. On voit déjà se développer à l'époque d'Edo des discours considérant les burakumin comme une autre ethnie que celle des Japonais. Ce seront néanmoins surtout la pénétration du darwinisme et de l'eugénisme au Japon, à la fin du XIX ${ }^{\mathrm{e}}$ siècle, qui donneront naissance à des discours racialistes et racistes au sujet des populations eta. Le terme de « race » (jinshu 人種), appliqué aux burakumin, est par ailleurs employé tel quel dans de nombreux écrits de la première moitié $\mathrm{du} \mathrm{Xx}^{\mathrm{e}}$ siècle. Nous avons développé ces points plus en détail lors des journées d'études du 22 janvier 2015 (« Populations japonaises », CEJ-Inalco) et du 24 mars 2016 (CEJ-Antenne de Toulouse).

150. Ibid., n ${ }^{\circ} 15$, le 10 novembre 1925. 
dans le Chikuhō, l'un des fondateurs du PCJ Yamakawa Hitoshi 山川均 (1880-1958) déclare en 1924 que l'idéal de la Suiheisha « ne peut se réaliser que grâce à l'entraide des trois mouvements d'émancipation des prolétaires, à savoir celui du syndicat ouvrier, du syndicat agricole et de la Suiheisha », soulignant qu' « ouvriers, métayers et burakumin ne forment qu'une seule et même classe, celle des opprimés. » Dans le même numéro, le leader du Syndicat des mineurs de l'ouest, Koyama Morito 小山盛人, affirme que « l'émancipation des buraku passe par l'émancipation économique, laquelle ne se réalise que grâce à une autre émancipation : celle de l'exploitation capitaliste par l'ensemble de la classe prolétaire ${ }^{151}$. $\gg$ Il s'agit de signifier que les luttes des burakumin ne sont que des variantes de celles du prolétariat, et qu'elles ont vocation à se retrouver au sein du PCJ. Mais le fait d'avoir pris la peine de rajouter les burakumin à côté des catégories classiques - paysannes et ouvrières - montre que la prise en compte du mouvement de la Suiheisha est difficilement évitable dans le contexte local.

En réalité, il semblerait que la Suiheisha du Kyūshū cherche surtout à tirer son épingle du jeu dans les luttes d'influence entre les tendances socialisantes et celles plus proches du PCJ. Lorsque l'influence de ce dernier faiblit, les revendications propres aux buraku ressurgissent : c'est le cas avec l'éphémère ${ }^{152}$ «Parti des paysans et des ouvriers » (Nōminrōdōtō 農民労働党, ci-dessous NRT), fondé en décembre 1925 à partir de la coalition entre la Suiheisha et différents syndicats. Au-delà des revendications spécifiques aux mines, son programme doctrinal (kōryō 綱領) consacre une attention particulière à la condition des burakumin dans les houillères, revendiquant « le même salaire pour le même travail, quels que soient le sexe, l'âge et la race [jinshu 人種] ${ }^{153}$. » Ce mot de « race » pourrait renvoyer aux ouvriers coréens, mais ceux-ci sont désignés dans le même texte par le terme de 《 peuple des colonies ( shokuminchi minzoku 植民地民族). Il s'agit donc des burakumin, d'autant que les militants de la Suiheisha les définissent eux-mêmes

151. Les propos de Yamakawa Hitoshi et Koyama Morito dans le premier numéro de Seibu sensen sont cités par SHINDō, 1978, p. 229.

152. Il dure à peine une journée avant d'être dissous par les autorités.

153. Programme doctrinal voté en 1925, cité par Tsuchiana, 1960, p. 256. 
comme un « peuple » minoritaire ${ }^{154}$, et que la Suiheisha était la seule association politique représentant une minorité au moment de la fondation de ce parti. On peut supposer que cette dernière a surtout pu se faire entendre en profitant des conflits intérieurs qui aboutiront, lors de la création du NRT, à la mise à l'écart de l'aile gauche communiste au profit des syndicats sociaux-démocrates. D'autant qu'à l'époque, la Suiheisha n'avait pas encore décidé de subordonner son combat contre les discriminations aux luttes de classes plus générales.

Le gouvernement, soupçonnant le NRT de liens avec le PCJ, ordonne sa dissolution dès sa fondation. Son successeur direct, le « Parti des ouvriers et des paysans » (Rōdōnōmintō 労働農民党, ci-dessous RNT), sera formé quelques mois plus tard, en mars 1926, mais il ne tient pas compte de la question buraku dans son programme doctrinal ${ }^{155}$. Lors de cette refondation, l'aile communiste a réussi à prendre le pouvoir. Ce nouveau rapport de forces se traduit par la subordination des causes des minorités discriminées à celles de la lutte des classes, alors même que le comité central du RNT compte dans ses rangs des cadres de la Suiheisha, comme Sakamoto Sei.ichirō 阪本清一郎 (1892-1987). Parallèlement, au sein des mouvements burakumin, l'influence du PCJ atteint son apogée et relègue le combat contre la discrimination anti-buraku derrière la lutte des classes.

\section{Le Taishū jih̄ō, de l'alliance sociale-démocrate au retour des communistes}

Cette année 1926 voit se multiplier les opérations antimilitaristes au sein de la Zenkyūsui, orchestrées par l'aile communiste de la ZKS et regroupées sous l'appellation « combat par accusation de discrimination du régiment de Fukuoka » (Fukuoka rentai sabetsu kyūdan tōso $\bar{o}$ 福岡連隊差別糾弹闘争). Pour protester contre la discrimination anti-buraku au sein du régiment militaire de

154. Ce terme de «peuple » (minzoku) est par exemple employé dans Zen Kyūshū Suineisha, 1985 [1925], no 15.

155. Telle est du moins l'analyse dominante parmi les chercheurs du centre de recherche Buraku Kaihō Jinken Kenkyūjo 部落解放人権研究所. YoshidA, 2013 ; TANAKA, 2001, p. 1098. Mais il faudrait analyser directement le programme doctrinal du Parti à la Bibliothèque de la Diète : Hososako, 1926. 
Fukuoka, la ZKS multiplie les boycottages et les occupations de leurs terrains d'entraînement, tout en dénonçant le militarisme avec le soutien de partis et de syndicats noyautés par des communistes, comme le RNT, ou la «Conférence des syndicats du travail du Japon » (Nihon rōdō kumiai hyōgikai 日本労働組 合評議会) ${ }^{156}$. Cette campagne de lutte contre le militarisme à Fukuoka se solde par l'arrestation en novembre 1926 de plusieurs cadres de la ZKS ${ }^{157}$, dont Matsumoto Ji.ichirō et Fujioka Shōuemon 藤岡正右衛門 (1892-1930), mais surtout de membres de l'aile bolchévique, tels Wada Tōsuke 和田藤助 ${ }^{158}$ (ZKS) et Kimura Kyōtarō 木村京太郎 (1902-1988) ${ }^{159}$. Ces répressions affaiblissent la Zenkyūsui, et le Suihei geppō finit par cesser de paraître après le numéro de juin 1927. Le journal renaît toutefois un an plus tard, en mai 1928, sous le titre de Taishū jihōo 大衆時報 (《Courrier du peuple ») ${ }^{160}$ : il est à présent devenu l'organe d'une coalition entre la Zenkyūsui et le «Syndicat des mineurs de Kyūshū »(Kyūshū tankōfu kumiai 九州炭坑夫組合, ci-dessous KTK). Ce syndicat, à tendance socialiste, est affilié au «Parti ouvrier et paysan du Japon » (Nihon Rōnōtō 日本労農党, ci-dessous « Nichirōtō ») $)^{161}$, puis après la dissolution de ce dernier, au «Parti social-démocrate populaire »(Shakai Minshūtō 社会民衆党).

L'alliance avec un syndicat de mineurs est naturelle : leur cause était déjà essentielle au Suihei geppō, édité au cœur des houillères du district de Kaho. En revanche, celle avec un syndicat socialiste, voire social-démocrate,

156. SHINDō, 1978, p. 234.

157. Pour le détail de ces arrestations, nous nous référons à ZeN Kyūshū SuIHEISHA, $1985, \mathrm{n}^{\circ} 26$, le $1^{\text {er }}$ mars 1927.

158. Haraguchi, 1988, p. 518.

159. Kimura prendra la carte du PCJ en 1927. Buraku Kaihō Jinken Kenkyūjo, 2001 p. 218.

160. Taishū jihō, no 3598,19281933 , reproduits dans Zen Kyūshū Suineisha, 1985 , p. 111-120.

161. Le Nihon Rōnōtō, socialiste, est différent des trois autres partis proches du PCJ appelés « Rōnōtō », à savoir le Rōdō Nōmintō (RNT, 1926-1928), le Rōdōsha Nōmintō 労働者農民党 (1928-1928) et le Rōnōtōo 労農党 (1929-1931). 
surprend d'autant plus que les cadres de la Zenkyūsui soutiennent dans le même temps le RNT, proche du PCJ. C'est ainsi que le chef de file de la ZKS, Matsumoto Ji.ichirō, et l'un des fondateurs de la Suiheisha au niveau national, Saikō Mankichi 西光万吉, se présentent aux élections nationales de février 1928 sous la houlette du RNT, respectivement à Fukuoka et Nara.

Les arrestations de 1926 ont causé une hémorragie parmi les communistes de la Zenkyūsui. Les éditeurs-rédacteurs du Suihei geppō réchappés des arrestations, Hanayama Kiyoshi et Tanaka Shōgetsu 田中松月 (1900-1993), sont d'autant plus proches des socialistes que l'éphémère NRT avait pleinement pris en compte la question spécifique des populations discriminées. Le Taish ū jihōo voit par ailleurs le jour dans un contexte où les mouvements communistes sont généralement affaiblis, notamment avec « l'affaire du 15 mars 1928 », qui voit l'arrestation de plusieurs mineurs communistes de la Zenkyūsui, dont Wada Hatsutarō 和田初太 郎, Sōmon Kotarō 惣門小太郎 et Matsumoto Kichinosuke ${ }^{162}$.

Au sein du comité de rédaction du Taishü jihō, les forces sont quantitativement équilibrées, avec cinq membres de la Suiheisha du district de Kaho, dont Hanayama Kiyoshi et Tanaka Shōgetsu, qui avaient fondé le Suihei geppō, et cinq membres du KTK, syndicat proche des socialistes ${ }^{163}$. Le rapport de force éditorial évoluera cependant en défaveur de la cause buraku au fil des publications, et ce, dès les premiers numéros ${ }^{164}$. Le titre même du journal révèle un certain degré de marginalisation de la Suiheisha face à la prédominance de la cause socialiste : Taish $\bar{u}$ jihō, que l'on peut traduire par « Courrier du peuple », dénote davantage l'intérêt général pour les masses exploitées que pour les minorités discriminées. Son premier numéro, daté du $1^{\text {er }}$ mai 1928 , est ainsi consacré à la journée des ouvriers, sans vraiment proposer d'articles en lien avec les buraku ou la Suiheisha.

162. Matsumoto, 1977, p. 107 ; Shindō, 1978, p. 237.

163. La composition du comité de rédaction est précisée dans ZEN KYūshū Suiheisha, 1985, numéro du 10 janvier 1929.

164. Par « les premiers numéros », nous désignons ceux publiés entre mai et août 1928, soient les numéros 35 à 38, puisque la numérotation de Suibei geppō est continuée par le nouveau journal. 
L'omniprésence du Nichirōtō est également frappante : alors qu'aucune publicité de la Suiheisha ne figure dans les premiers numéros, plusieurs publicités du Nichirōtō sont en revanche publiées, dont celle de la section Nichirōtō du district de Kaho, celle des bureaux locaux du « Journal du Nichirōtō » dans les districts de Kaho et de Tagawa, et celle d'un candidat officiel de ce même parti aux élections locales ${ }^{165}$. La mention de la Suiheisha, lorsqu'elle apparait, est toujours donnée en plus petits caractères que pour le Nichirōtō. Même le syndicat KTK, qui représente pourtant la moitié du comité de rédaction, occupe moins de place que ce parti. Quelques mineurs sont mentionnés, mais souvent dans des rubriques en petits caractères. De même, si un candidat aux élections exerce la profession de mineur, sa mine d'appartenance est mentionnée de façon moins visible que celle du Nichirōtō ${ }^{166}$. Ceci s'explique sans doute par le fait que le KTK avait alors particulièrement besoin de soutien politique : il s'était en effet retrouvé affaibli après la scission de la «Confédération générale du travail du Japon » (Nihon rōdō sōdōmei 日本労働総同盟) ${ }^{167}$, à laquelle il appartenait, et qui allait se diviser entre la Conférence des syndicats du travail du Japon, établie en mai 1925, et la «Ligue des syndicats du travail du Japon » (Nihon rōdō kumiai dōmei 日本労働 組合同盟), fondée en décembre de l'année suivante.

À partir du numéro de décembre 1928, le rapport de force évolue à nouveau, avec une première page pleinement consacrée aux préoccupations de la Suiheisha. À côté des procès de l'affaire du régiment de Fukuoka, en cours d'instruction, les méthodes de 《lutte par accusation publique » (kyz̄dan tōsō 紏弾闘争) des discriminations anti-buraku, dont l'organisation est coutumière, occupent l'autre partie de la une. Celle-ci dénonce en l'occurrence l'emploi du mot eta par un yakuza de Nagasaki proche du pouvoir local, Fujita Matao 藤田又雄, accusé de « voyou réactionnaire » pour avoir déclaré : « appeler eta ceux qui sont des eta, où est le problème ${ }^{168}$ ? » À l'inverse, le Nichirōtō, omniprésent jusqu'au numéro précédent, est quasiment absent des articles de décembre 1928. Ce parti est

165. Taish ū jihō du $1^{\text {er }}$ mai 1928 , et du $1^{\text {er }}$ août 1928 , ibid.

166. Ibid., numéro du $1^{\mathrm{er}}$ août 1928.

167. Différent de la Zen-Nihon rōdō sōdōmei 全日本労働総同盟.

168. Taishū jihō, Ibid., nº 42,1 $1^{\mathrm{er}}$ décembre 1928. 


\section{CIPANGO}

110 Cahiers d'études japonaises $n^{\circ} 23$

effectivement en train de disparaître pour se fondre, avec certains autres partis de gauche, dans la coalition du «Parti du peuple du Japon » (Nihon Taishūtō 日本 大衆党), qui se constituera vingt jours après la publication de ce numéro. On peut se demander si les membres de la Zenkyūsui n’ont pas profité de cette période de flottement pour faire paraître leurs revendications en faveur des buraku comme du temps du Suihei geppō, d'autant que les rédacteurs principaux de Taish ū jihō ne s'allieront finalement pas à ce nouveau parti de coalition, mais à un parti plus centriste. Ainsi, aux élections départementales de janvier 1929, Hanayama Kiyoshi se présente comme auparavant avec le soutien du KTK, mais aussi celui du Parti social-démocrate populaire. Le numéro du 10 janvier 1929 du Taishū jihō affiche d'ailleurs un encart de soutien de Miyachika Kōji 宮近綱次, un élu de ce même parti.

Par la suite, la parution du Taish $\bar{u}$ jihō devient très espacée, à peine un numéro par an. Il faudra attendre 1933 pour que son rythme de parution redevienne plus régulier, ce qui correspond au moment où le journal se rapproche d'un nouveau syndicat de mineurs du Kyūshū, le « Syndicat du travail des houillères de l'Ouest » (Seibu kōzan rōdō kumiai 西部鉱山労働組合, ci-dessous SKRK $)^{169}$. Ce dernier est proche du « Conseil national des syndicats du travail du Japon » (Nihon rōdō kumiai zenkoku kyōgikai 日本労働組合全国協議会, ci-dessous Zenkyō 全協), aux mains des communistes, et rival du syndicat social-démocrate auquel le Taishū jihō s'était allié précédemment. En janvier 1934, le Taishū jihōo devient même l'organe officiel du SKRK. Le rapprochement avec ce syndicat affilié au PCJ est cependant plus ancien : il date au moins de l'année 1931, lorsque Hanayama Kiyoshi s'était présenté aux élections départementales de Fukuoka avec le soutien officiel du SKRK, en plus de celui du « Parti national du peuple ouvrier et paysan » (Zenkoku rōnō taishūtō 全国労農大衆党). 1931 fut par ailleurs une année de combats dans les mines du Chikuhō (Chikuhō tanden sōgi 筑豊炭田争議) ${ }^{170}$, dont les grévistes furent soutenus par le SKRK, le Zenkyō, et bien sûr, la Zenkyūsui.

169. Le Taishū jihō devient l'organe du SKRK en 1934 (nº99-107). Ces numéros sont reproduits dans ZEN Kyūshū SuIHeIsha, 1985, p. 121-130.

170. Matsumoto, 1977, p. 104-115 ; Shindō, 1978, p. 238. 
Le premier numéro du Taish ū jihō en tant qu'organe du SKRK, daté de janvier $1934^{171}$, pose le syndicat en représentant des mineurs du Chikuhō, toujours en pleine lutte de classes. L'encart de la première page annonce ainsi en gros caractères :

Encore une explosion à la mine d'Akaike. [...] Faisons condamner à mort les responsables et les capitalistes qui, en cherchant avidement le profit, ont massacré de nombreux mineurs, et font ainsi vivre leur famille dans une haine sans fin !! Dédommagements à vie aux familles des défunts ${ }^{172}$ !!

La majeure partie des articles de ce numéro s'adressent aux travailleurs de ces houillères : ils évoquent «l'ennemi commun des cent mille mineurs du Chikuhō » (p. 2), et incitent les lecteurs à exprimer leurs difficultés ou attentes dans une rubrique intitulée «Le bras des mineurs » (p. 4). Des conseils de lecture sont également dispensés pour éduquer les masses : « Lis !!! Les livres sont notre pain quotidien », avise ainsi une colonne recommandant trois auteurs, dont Marx et Yamakawa Hitoshi. Dans ces articles à l'attention des mineurs du Chikuhō, il n'est en revanche jamais fait mention de la condition des mineurs buraku. Hanayama Kiyoshi, qui est toujours l'éditeur et le directeur de Taishù jihō, ne mentionne pas non plus la question buraku dans les deux articles qu'il écrit pour ce premier numéro. Un seul texte émane de la Suiheisha, sous la plume de Matsumoto Ji.ichirō, alors président de son comité central. Inséré dans le coin inférieur d'une page, il s'agit seulement d'une carte de vœux pour le nouvel an, qui rapporte en petits caractères quelques nouvelles concernant la Suiheisha.

Il faut attendre la publication du numéro spécial d'avril 1934 pour voir abordée la question de la condition burakumin. Ce numéro spécial « Suiheisha » du Taish $\bar{u}$ jihō est très différent des anciens numéros de Taishū jihōou de Suibei geppō. On n'y retrouve ni accusations publiques, ni lettres d'excuses ou de soutiens directs à la ZKS, mais en lieu et place, des récits didactiques rapportant des cas de discrimination, notamment à l'école. Comme le montre la préface à ces récits, il

171. Taishū jihō, Zen Kyūshū Suineisha, 1985, nº 99, le $1^{\text {er }}$ janvier 1934.

172. Ibid. 


\section{CIPANGO}

112 Cahiers d'études japonaises $n^{\circ} 23$

s'agit de sensibiliser les lecteurs aux difficultés particulières que rencontrent les burakumin et d'expliquer les raisons de certaines pratiques de la Suiheisha :

Du point de vue des gens ordinaires, les actions de la Suiheisha, telles ses accusations d'actes discriminatoires, doivent paraître incompréhensibles. [...] Vous expliquer à quel point un seul mot suffit pour nous blesser irrémédiablement ne suffit pas à obtenir votre compréhension. Ainsi, nous nous permettons de vous raconter ici une histoire personnelle $[\ldots]^{173}$.

À la fin de l'article, l'auteur insiste dans son effort de sensibilisation :

Vous pensez sans doute qu'aujourd'hui, en ces jours de l'ère Shōwa, il ne doit plus y avoir de discriminations de ce genre. [...] Mais c'est précisément parce que ce type de discriminations existe toujours que la Suiheisha se voit obligée de poursuivre sa pratique de l'accusation publique ${ }^{174}$.

Sensibiliser les lecteurs aux pratiques accusatoires de la Suiheisha est d'autant plus important que les camarades des mines sont parfois les auteurs de ces discriminations. En l'état actuel de nos recherches, nous ne pouvons affirmer si ce numéro est une concession arrachée par les membres de la Zenkyūsui, ou si le SKRK, donc les communistes, ressentent au cours de l'année $1934^{175}$ le besoin, local ou national, de resserrer les rangs et de s'adjoindre plus fortement le soutien de la Suiheisha et de l'ensemble des burakumin.

Par ailleurs, ce numéro spécial « Suiheisha » n’évoque aucunement la question des mineurs, ni des ouvriers en général, comme si la condition des minorités discriminées et celle des prolétaires étaient désormais deux réalités différentes. Une évolution de taille par rapport aux discours assimilationnistes du PCJ et de

173. Ibid., $\mathrm{n}^{\circ}$ 102, le 10 avril 1934.

174. Ibid.

175. Moment où les mouvements communistes sont affaiblis par la répression et les conversions politiques successives. 
Sano Manabu, qui dix ans plus tôt, considérait le Chikuhō et ses houillères comme le lieu idéal pour parvenir à la solidarité entre ouvriers buraku et non buraku ${ }^{176}$.

\section{Conclusion}

Dans le bassin du Chikuhō, le lien entre eta et charbon, puis entre buraku et houillères, s'avère ancien. Il procède de facteurs à la fois historiques et sociaux, issus des stratégies locales des dirigeants des fiefs de l'époque Edo, associés à des facteurs plus pédologiques de localisation des gisements de charbon.

Avec l'industrialisation et la constitution d'un prolétariat minier, ce lien s'est mécaniquement atténué, surtout durant la période d'expansion des houillères, à la fin du XIX ${ }^{\mathrm{e}}$ siècle. Mais à partir des années 1920, puis au cours de l'aprèsguerre, la présence buraku au sein des mines se renforce de nouveau. On peut se demander si certains historiens spécialistes des buraku n'en sont pas venus à lier aussi étroitement charbon et buraku pour cette raison précise, et à extrapoler, ce faisant, la situation du Chikuhō. Du point de vue des buraku, la réciproque est plus certaine : le charbon semble être resté pour les communautés buraku du Chikuhō un élément déterminant en termes d'emploi, de revenus et même de mémoire collective.

En effet, la formation du prolétariat industriel, auquel les burakumin furent intégrés dès les débuts, n’a pas gommé la discrimination ancestrale dont ils faisaient l'objet avant Meiji : ségrégation dans l'habitat, relégation vers les tâches les moins qualifiées, les moins rémunérées, et même maintien des tabous, comme les interdictions d'accès à l'intérieur des fosses.

Les grands groupes miniers tels que Mitsui, Mitsubishi ou Sumitomo ont adopté des stratégies différentes vis-à-vis de ces communautés, entre évitement et dissimulation. Mais les groupes plus locaux, comme celui d'Asō Takichi, qui avait une bonne connaissance du Chikuhō et de ses buraku, ont plus certainement développé une stratégie assez claire : celle de tirer au maximum profit de cette main-d'œuvre disponible, moins chère et plus captive. Une logique qui voit son

176. SANO, 1923, et 《Suihei shakai no kensetsu 》水平社会の建設, in ZEN KYŪSHŪ SuIHEISHA, 1985, nº 3, août 1924. 


\section{CIPANGO}

114 Cahiers d'études japonaises $n^{\circ} 23$

prolongement avec l'usage privilégié de la main-d'œuvre coloniale chez Asō dans les années 1930, pour des salaires souvent plus bas encore que ceux des burakumin, et des conditions de travail souvent difficiles.

Au sein des mouvements ouvriers, et en particulier du PCJ, le plus proche allié de la Suiheisha, la question du traitement des discriminés est restée à géométrie variable. L'analyse théorique de la lutte des classes peut en effet aussi bien conduire à les intégrer dans son dispositif qu'à les considérer comme une préoccupation secondaire. L'état du rapport de force entre le PCJ et la Suiheisha, répercuté sur les houillères du Chikuhō par la présence de la Zenkyūsui et des syndicats affiliés au PCJ, se montre ici déterminant : quand les temps sont durs, le besoin de soutien chez les uns ou les autres conduit les membres de la ZKS à insister moins fortement sur la particularité des buraku, et les responsables du PCJ à conscientiser leurs militants sur la justesse du combat contre la ségrégation anti-buraku.

Si l'après-guerre voit la fin progressive des mines du Chikuhō, le taux de mineurs burakumin semble paradoxalement avoir augmenté à mesure que fermaient ces houillères. Le faible niveau de qualification de ces populations, qui rendait la reconversion vers d'autres secteurs moins aisée que pour les salariés non burakumin, n'est certainement pas sans lien avec ce phénomène.

On pourrait penser qu'avec la patrimonialisation actuelle des mines de cette région, que ce soit sous la forme de musées, d'expositions ou de monuments commémoratifs, une mémoire de la présence buraku aurait pu voir le jour. En réalité, un grand nombre d'anciens puits sont situés à proximité de buraku, et toujours peuplés de burakumin ${ }^{177}$. Comme l'identification et la localisation de ces hameaux restent aujourd'hui l'enjeu majeur de la lutte des associations de défense des buraku à Kyūshū, mais aussi dans le reste du Japon, les groupes issus de la ZKS s'opposent à toute mention sur le terrain, ou dans des publications, qui permettrait de localiser précisément les buraku du Chikuhō.

177. D'après le dernier recensement national de 1993, le département de Fukuoka est aujourd'hui le deuxième département le plus peuplé par les populations burakumin, après celui de Hyōgo. Sōmuchō, 1995, p. 72. 


\section{Bibliographie indicative}

Aso Tatsuo 安蘓龍生, 1979, «Bakuhan jidai no tagawa ni okeru sekitan saikutsu to hisabetsu buraku 》幕藩時代の田川における石炭採掘と被差別部落 [L'extraction de la houille et les buraku discriminés à Tagawa durant l'époque féodale] in Buraku kaihōshi: Fukuoka 部落解放史・ふくおか [Histoire de l'émancipation des buraku: Fukuoka], nº 15, pp. 56-85.

BURAKU KAIHŌ KENKYŪJO 部落解放研究所 (ed.), 1981, Buraku mondai 部 落問題 [Questions buraku], Kaihō shuppansha 解放出版社, Ōsaka.

BURAKU KAIHŌ JINKEN KENKYūjo 部落解放人権研究所 (ed.), 1981, Buraku jinken jiten 部落問題 - 人権事典 [Dictionnaire encyclopédique de la question buraku et des droits de l'Homme], Kaihō shuppansha 解放出版 社, Ōsaka.

Chikuhō Sekitan KōgYōshi Nenpyō Hensan IInKai 筑豊石炭砿業史 年表編纂委員会編 (ed.), 1973, Chikubō sekitan kōgyōshi nenpyō 筑豊石 炭鉱業史年表 [Chronologie de l'histoire industrielle du charbon dans le Chikuhō], Tagawa kyōdo kenkyūkai 田川郷土研究会, Tagawa.

Chūō YŪWA Jigyō KYōKaI 中央融和事業協会, 1970 [1926-1941], Yūwa jigyō nenkan 融和事業年鑑 [Annuaire des œuvres yüwa 1926-1941], Buraku Kaihō Kenkyūjo, Ōsaka.

Chūō YŪWA Jigyō KYōKaI 中央融和事業協会, 1974, Yūwa jigyō kenkyū 融 和事業研究 [Recherches sur les œuvres yūwa], réédition des quatre-vingt numéros, Sekai Bunko 世界文庫, Tōkyō.

ENDō Masao 遠藤正男, 1942, Kyūshū keizaishi kenkyū 九州経済史研究 [Recherches sur l'histoire économique du Kyūshū], Nihon hyōronsha 日本 評論社, Tōkyō, 331 p. 
CIPANGO

116 Cahiers d'études japonaises $\mathrm{n}^{\circ} 23$

FukUoka BURAKUShi KenkyŪKAI 福岡部落史研究会 (dir.), 1979, Fukuokaken hisabetsu burakushi no shosō 福岡県被差別部落史の諸相 [Divers aspects de l'histoire des buraku discriminés du département de Fukuoka], Fukuoka burakushi kenkyūkai 福岡部落史研究会, Fukuoka, 441 p.

FUKUOKA BURAKUSHI KENKYŪKAI 福岡部落史研究会 (dir.), 1989, Fukuoka no buraku kaihōshi 福岡の部落解放史 [Histoire de l'émancipation des buraku de Fukuoka], tome 2, Kaichōsha 海鳥社, Fukuoka, 263 p.

HARADA Tomohiko 原田伴彦, 1975 [1973], Hisabetsu buraku no rekishi 被差 別部落の歴史 [Histoire des hameaux discriminés], Asahi shinbunsha 朝日 新聞社, Tōkyō, 396 p.

HaraguCHi Eiyū 原口穎雄, 1988, « Kikanshi “Suihei geppō” ni miru Zenkyūshū Suiheisha no undō » 機関紙『水平月報』にみる全九州 水平社の運動 [L'activité de la Zenkyūshū Suiheisha au regard de l'organe Suihei geppō], in SEINAN CHI.IKISHI KenkyŪKaI 西南地域史研究 会 (ed.), Seinan chi.iki no shiteki tenkai: kindai-hen 西南地域の史的展開 一近代篇 [Développement historique des régions du Sud-Ouest à l'époque de la modernisation], Shibunkaku shuppan 思文閣出版, Kyōto.

Haraguchi Eiyū 原口頴雄, 1989, 《Suiheisha no shisō to undō »水 平社の思想と運動 (Idéologies et mouvements de la Suiheisha), in FukUOKa BURAKUshi KenkyūKaI 福岡部落史研究会 (ed.), Fukuoka no buraku kaihōshi 福岡の部落解放史, Kaichōsha 海鳥社, Fukuoka.

Haraguchi Eiyū 原口穎雄, 2001 in Buraku Kaihō Jinken Kenkyūjo 部落解放人権研究所 (ed.), Buraku mondai jinken jiten 部落問題 · 人権 事典 [Dictionnaire encyclopédique de la question buraku et des droits de l'Homme], Kaihō shuppansha, Ōsaka.

Hososako Kanemitsu 細迫兼光, 1926, Rōdōnōmintō no kōryō to seisaku 労働 農民党の綱領と政策 [Programme doctrinal et mesures proposées par le Rōdōnōmintō], Rōdōnōmintō Chōsabu 労働農民党調査部, Tōkyō. 
KAneko Uzeki 金子雨石, 1975, « Mitsui Tagawa tankō ni okeru rōdōsha no boshū to kaiko, 1 »三井田川炭鉱における労働者の募集と解雇, 1 [Recrutement et licenciement des ouvriers dans les mines Mitsui de Tagawa, 1] in ENERUgî-ShI KenKyūKaI エネルギー史研究会 (ed.), Enerugî-shi kenkyū nōto エネルギー史研究ノート [Cahier de recherches sur l'histoire de l'énergie], $\mathrm{n}^{\circ} 5$.

KAWAMUKAI Hidetake 川向秀武, 1989, « Buraku kaizen to seikatsu kadai 》 部落改善と生活課題 [Améliorations des buraku et problèmes de la vie quotidienne], in FuKUOKA BURAKUshi KENKYŪKAI 福岡部落史研究 会 (ed.), Fukuoka no buraku kaihōshi 福岡の部落解放史 [Histoire de l'émancipation des buraku de Fukuoka], Kaichōsha 海鳥社, Fukuoka.

Kindai burakushi shiryō shüsei 近代部落史資料集成 [Compilation de documents historiques sur les buraku à l'époque de la modernisation], 1984-1987, San.ichi shobō 三一書房, Tōkyō.

Kyūshū Daigaku Sekitan Kenkyū Shiryō Sentā 九州大学石炭研究 資料センター (dir.), 1993, Sekitan kenkyū shiryō sōsho 石炭研究資料叢 書 [Collection de documents historiques pour les recherches sur le charbon], vol. 14, Kyūshū daigaku sekitan kenkyū shiryō sentā 九州大学石炭研究資 料センター, Fukuoka, 217 p.

Kyūshū Daigaku Sekitan Kenkyū Shiryō Sentā 九州大学石炭研究 資料センター (dir.), 2000, Sekitan kenkyū shiryō sōsho 石炭研究資料叢 書 [Collection de documents historiques pour les recherches sur le charbon], vol. 21, Kyūshū daigaku sekitan kenkyū shiryō sentā 九州大学石炭研究資 料センター, Fukuoka, 215 p.

MAHARA Tetsuo 馬原鉄男, 1973, Nihon shihon shugi to buraku mondai 日本資 本主義と部落問題 [Le capitalisme japonais et la question buraku], Buraku mondai kenkyūjo 部落問題研究所, Kyōto, 493 p. 


\section{CIPANGO}

118 Cahiers d'études japonaises $\mathrm{n}^{\circ} 23$

Matsumoto Kichinosuke 松本吉之助, 1977, Chikuhō ni ikiru 筑豊に生きる [Vivre dans le Chikuhō], Buraku mondai kenkyūjo, Kyōto.

MatsuZaki Taketoshi 松崎武俊, 1979, «Edoki ni okeru fukuoka chihō no buraku to nōgyō, sono 5 » 江戸期における福岡地方の部落と農業, そ の五 (Les buraku et l'agriculture dans la région de Fukuoka à l'époque Edo, $\mathrm{n}^{\circ}$ 5), in Fukuoka-ken hisabetsu burakushi no shosō 福岡県被差別部落史の 諸相 [Divers aspects de l'histoire des buraku discriminés du département de Fukuoka], Fukuoka burakushi kenkyūkai 福岡部落史研究会, Fukuoka, pp. $85-120$.

NAGAsue Toshio 永末十四雄, 1973, Chikuhō : sekitan no chi.ikishi 筑豊一石 炭の地域史 [Chikuhō: une histoire régionale du charbon], Nihon hōsō shuppan kyōkai 日本放送出版協会, Tōkyō, 248 p.

NaGasue Toshio 永末十四雄, 1979, « Chikuhō o chūshin to shita shihon shugi no hattatsu to buraku mondai » 筑豊を中心とした資本主義の発達と部 落問題 [Le développement du capitalisme et la question buraku à partir du Chikuhō] in Buraku kaihōshi: Fukuoka 部落解放史・ふくおか[Histoire de l'émancipation des buraku: Fukuoka], nº 15.

NAGAsue Toshio 永末十四雄, 1989, «Sekitan kōgyō to buraku mondai »石 炭鉱業と部落問題 [L'industrie charbonnière et la question buraku], in Funuoka BuRAKushi KenkyŪKai (ed.), Fukuoka no buraku kaihōshi $i$ 福岡 の部落解放史, [Histoire de l'émancipation des buraku de Fukuoka] tome 2, Kaichōsha 海鳥社, Fukuoka, pp. 69-106.

NAImushō 内務省 [ministère de l'Intérieur], 2000 [1924], Kōgyō rōdō jijō chōsho 鉱業労働事情調書 [Enquête sur la situation du travail minier], in Kyūshū Daigaku Sekitan Kenkyū Shiryō Sentā 九州大学石炭研 究資料センター (ed.), Sekitan kenkyū shiryō sōsho 石炭研究資料叢書 [Collection de documents historiques pour les recherches sur le charbon], vol. 21, Kyūshū daigaku sekitan kenkyū shiryō sentā 九州大学石炭研究資 料センター, Fukuoka, 215 p. 
Nakamura Naofumi 中村尚史, 2008, 《“Chihō zaibatsu” no tanjō », in ISS Discussion Paper Series, Tōkyō daigaku shakaikagaku kenkyūjo 東京大学社 会科学研究所, Tōkyō.

OGINo Yoshihiro 荻野喜弘, 1993, Chikuhō tankō rōshi kankei-shi 筑豊炭鉱労 使関係史 [Une histoire des relations de travail dans les mines du Chikuhō], Kyūshū daigaku shuppankai 九州大学出版会, Fukuoka, 448 p.

Sabouret Jean-François, 1983, L'Autre Japon: les burakumin, Maspero, Paris, $155 \mathrm{p}$.

Sakamoto Yūichi 坂本悠一, 1997, « Chikuhō sekitan kōgyō to hisabetsu buraku »筑豊石炭鉱業と被差別部落 [L'industrie houillère du Chikuhō et les buraku discriminés] in Buraku mondai kenkyū 部落問題研究 [Études de la question buraku], n 140 .

SANo Manabu 佐野学, 1923, Suihei undōo 水平運動 [Mouvement pour l'égalité], archives personnelles de l'auteur, $29 \mathrm{p}$.

SHINDŌ Toyo.o 新藤東洋男, 1978, Chikuhō no jokōfutachi 筑豊の女坑夫た $ち$ [Les femmes mineurs du Chikuhō], Buraku mondai kenkyūjo 部落問題 研究所, Kyōto.

Sōmuchō 総務庁 [Agence des Affaires, 1995, Dōwa mondai no genkyō, heisei nananenban 同和問題の現況平成七年版 [État actuel de la question dōwa, année 1995], Chūō hōki shuppan 中央法規出版, Tōkyō.

Sumrya Mikio 隅谷三喜男, 2003 [1968], « Nihon sekitan sangyō bunseki »日 本石炭産業分析 [Analyse de l'industrie minière au Japon] in Sumiya Mikio chosakushū 隅谷三喜男著作集 [Recueil des travaux de Sumiya Mikio], vol. 4, Iwanami shoten 岩波書店, Tōkyō. 


\section{CIPANGO}

120 Cahiers d'études japonaises $n^{\circ} 23$

TACHIBANA Takashi 立花隆, 1983 [1978], Nihon kyōsantō no kenkyū 日本共産 党の研究 [Recherches sur le Parti communiste japonais], vol. 3, Kōdansha 講 談社, Tōkyō, 362 p.

TAKano Nobuharu 高野信治, 2007, « Kinsei daimyō no nōsei tenkai to shakai sabetsu »近世大名の農政展開と社会差別 [Politiques agricoles et discrimination sociale sous l'égide des seigneurs de la période moderne] in Hikaku shakai bunka 比較社会文化 [Social and Cultural Studies], vol. 13, pp. 36-43.

TAKI.I Yoshitaka 滝井義高, 1985, «Assatsu sareta tankō »圧殺された炭鉱 [Houillères étouffées], in Isomura Ei.ichi 磯村英一 (ed.), Teihen shakai 底 辺社会 [La société des bas-fonds], Yūzankaku shuppan 雄山閣出版, Tōkyō, pp. 203-216.

TANAKA Masato 田中真人, 2001, « Rōdōnōmintō » 労働農民党, in BURAKU KAIHŌ JINKEN KENKYŪJO 部落解放人権研究所 (ed.), Buraku mondai jinken jiten 部落問題・人権事典, Kaihō shuppansha, Ōsaka.

TokIta Yoshihisa 戸木田嘉久, 1976, Hatarakumono to buraku mondai 働< ものと部落問題 [Les travailleurs et la question buraku], Buraku mondai kenkyūjo shuppanbu 部落問題研究所出版部, Kyōto.

ToKIta Yoshihisa 戸木田嘉久, 1989, Kyūshū tankō rōdō chōsa shūsei 九州炭 鉱労働調査集成 [Compilation d'enquêtes sur le travail dans les mines de Kyūshū], Hōritsu bunkasha 法律文化社, Kyōto, 427 p.

Tokita Yoshihisa 戸木田嘉久, 1992, « Chikuhō no tankō to buraku no koto » [À propos des mines et des buraku du Chikuhō] in Ritsumeikan keizaigaku 立 命館経済学 [Sciences économiques de Ritsumeikan], nº 5, vol. 41.

Tōkyō Chinō Shokugyō Shōkai Jimukyoku 東京地方職業紹介事務局 [Bureau de l'emploi de Tōkyō], 2000 [1928], « Chikuhō o chūshin to shita shihon shugi no hattatsu to buraku mondai » 最近内地在留朝鮮人調べ, 
in Sekitan kenkyū shiryō sōsho 石炭研究資料叢書 [Collection de documents historiques pour les recherches sur le charbon], vol. 21, Kyūshū daigaku sekitan kenkyū shiryō sentā 九州大学石炭研究資料センター, Fukuoka.

Tsuchiana Fumito 土穴文人, 1975, « “Nōminrōdōtō” no seiritsu keika to rōdōkumiai 》「農民労働党」の成立経過と労働組合 [Le processus de formation du « Nōminrōdōtō » et les syndicats ouvriers] in Hōsei DAIGAKU ShaKAIgaKUBU GAKKaI 法政大学社会学部学会 (ed.), Shakai rōdo kenkyū 社会労働研究 [Recherches sur le travail et la question sociale], $\mathrm{n}^{\circ}$ 12, pp. 195-271.

TsūSHō SANGYōSHō 全九州水平社通商産業省 [ministère du Commerce, 1964, Honpō kōgyōno sūsei gojūnen-shi, zokuhen 本邦鉱業の趨勢 50 年史, 続篇 [Histoire d'un demi-siècle de l'industrie houillère de notre pays], Tsūshō sangyō chōsakai 通商産業調査会, Tōkyō.

UEDA Masayo うえだまさよ, 1977, 《Watashi no oitachi »わたしのおい たち [Mon enfance], in Tōjō Takashi 東上高志 (ed.), Watasha soredemo ikitekita わたしゃそれでも生きてきた [Pourtant, j'ai continué à vivre], Buraku mondai kenkyūjo, Kyōto.

YADA Toshifumi 矢田俊文, 2014, Yada Toshifumi chosakushū 矢田俊文著作集 [OEuvres de Yada Toshifumi], vol. 1, Hara shobō 原書房, Tōkyō.

YoshidA Fumiyoshi 吉田文茂, 2013, 《 Musan seitō kessei to zenkoku suiheisha 》無産政党結成と全国水平社, publié sur la page internet du centre de recherche Buraku Kaihō Jinken Kenkyūjo 部落解放人権研究所.

UENO Eishin 上野英信, 1960, Owareyuku kōfutachi 追われゆく坑夫たち [Les mineurs traqués], Iwanami shoten 岩波書店, Tōkyō, 246 p.

ZeN Kyūshū SuineIsha 全九州水平社, 1985, Suihei geppō 水平月報 [Bulletin mensuel de la Suiheisha de Kyūshū], nº 1-28, 1924-1927 ; archives 


\section{CIPANGO}

122 Cahiers d'études japonaises $n^{\circ} 23$

reproduites dans Suibei geppō fukkokuban 水平月報復刻版, Fukuoka burakushi kenkyūkai 福岡部落史研究会, Fukuoka, p. 1-109.

Résumé : la région du Chikuhō (Kyūshū) reste fortement associée à l'histoire $\mathrm{du}$ charbon. Les liens entre communautés de parias (burakumin) et l'industrie de la houille y sont moins connus, l'historiographie d'après-guerre ayant peu abordé cette question. Les communautés buraku sont pourtant bien présentes dans le Chikuhō, le plus souvent à proximité des anciennes mines. Nous montrons ainsi qu'il existe un lien organique, entre la présence buraku dans le Chikuhō et l'industrie du charbon, procédant de facteurs historiques et sociaux. L'industrialisation des houillères à la fin $\mathrm{du} \mathrm{XIX}^{\mathrm{e}}$ siècle aurait pu conduire à l'intégration de ces burakumin au sein de la classe ouvrière émergente. Mais la discrimination a pourtant perduré au sein du prolétariat minier et fit même partie des stratégies de certains groupes industriels pour la gestion des populations ouvrières. Cette question de la spécificité buraku s'est aussi posée au mouvement ouvrier, au sein des syndicats et dans les stratégies du Parti communiste japonais. La nature et l'évolution du rapport des groupes de défense des burakumin aux autres syndicats de mineurs ont ainsi évolué, selon les impératifs du moment et les rapports de force, entre solidarité, alliance et subordination.

Mots-clés : Charbon, parias, buraku, Chikuhō, Parti communiste, discrimination, populations ouvrières

Abstract: The region of Chikuhō (Kyūshü) remains strongly associated with a long history of coal mining. The links between outcast communities (burakumin) and the coal industry are less well known, as post-war historiography has hardly broached the subject. However, buraku communities are well represented in Chikuhō, most often in the vicinity of former mines. We show that there is an organic link, between the buraku presence in Chikuhō and the coal industry, stemming from historical and social factors. The industrialization of the coal mines at the end of the 19th century could have led to the integration of these burakumin into the emerging working class. But discrimination persisted within the mining proletariat and was even part 
of the strategies of some industrial groups for the management of the working-class populations. This question of buraku specificity has also been raised within the workers' movement, the labor unions and in the strategies of the Japanese Communist Party. The nature and evolution of the relationship between burakumin defense groups and other miners' unions has thus evolved, in accordance with punctual imperatives and the balance of power, between solidarity, alliance and subordination.

Keywords: Coal, Outcasts, buraku, Chikuhō, Japan Communist Party, discrimination, working-class

キーワード：筑豊炭田、部落問題、労資関係、就職差別、労働運 動 
